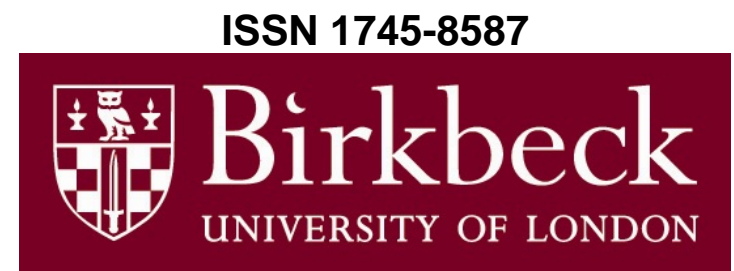

School of Economics, Mathematics and Statistics

BWPEF 0508

\title{
Dynamic Hedging of Financial Instruments When the Underlying Follows a Non-Gaussian Process
}

\author{
Álvaro Cartea
}

May 2005 


\title{
Dynamic Hedging of Financial Instruments When the Underlying Follows a Non-Gaussian Process
}

\author{
Álvaro Cartea*
}

May 25, 2005

\begin{abstract}
Traditional dynamic hedging strategies are based on local information (ie Delta and Gamma) of the financial instruments to be hedged. We propose a new dynamic hedging strategy that employs non-local information and compare the profit and loss (P\&L) resulting from hedging vanilla options when the classical approach of Delta- and Gammaneutrality is employed, to the results delivered by what we label Delta- and FractionalGamma-hedging. For specific cases, such as the FMLS of Carr and Wu (2003a) and Merton's Jump-Diffusion model, the volatility of the P\&L is considerably lower (in some cases only 25\%) than that resulting from Delta- and Gamma-neutrality.
\end{abstract}

\footnotetext{
*Birkbeck College, University of London. Email: a.cartea@bbk.ac.uk. This work has been supported by the Nuffield Foundation. The author is grateful for comments from seminar participants at the University of Toronto, New York University, Fields Institute Toronto, Federal Reserve Board Washington D.C., Goldman Sachs New York, CUNY Baruch College, Columbia University, Bachelier Finance Society 2004 meeting, Univerisdad Nacional Autónoma de México, King's College London, Judge Institute University of Cambridge, the OxfordPrinceton Mathematical Finance 2004 meeting, ESSEC, Katholieke Universiteit Leuven, Illinois Institute of Technology and Heriot-Watt University.
} 
Since the seminal work of Black and Scholes (1973), a great deal of effort has been expended on proposing new models to describe the dynamics of securities under both the riskneutral and statistical measures. These models include stochastic volatility or time-changed models, (Heston (1993), Stein and Stein (1991), Carr and Wu (2004)); jump-diffusion models (Merton (1990), Kou (2002)); and more general jump processes (Madan and Seneta (1990), Carr, Geman, Madan, and Yor (2002), Carr and Wu (2003a)).

Non-Gaussian models such as those mentioned above may be very versatile at capturing some of the main characteristics of the distribution of financial securities, including skewness, heavy tails and correlation. However, although these are characteristics that any model must take into consideration, there are also the questions of how to price financial instruments written on an underlying that follows one of these models and how to estimate the relevant parameters under both the physical and risk-neutral measures. It is well-known that for the majority of non-Gaussian models there is no unique equivalent martingale measure (EMM) under which pricing is performed; exceptions include the fixed jump size Poisson model. Therefore proposed models must not only look at the range of EMM's arising from them, but must also consider how a particular one is chosen, Carr and $\mathrm{Wu}(2003 \mathrm{~b})$.

Although the pricing of derivative instruments is key in financial modelling, the hedging of instruments is at least as important. Large market players or market makers are responsible for the liquidity of instruments that are traded on a regular basis in the different exchanges but they also trade most over-the-counter instruments such as tailor-made instruments, exotic options, and other less liquid assets. Critical to the liquidity or availability of these instruments is the ability to hedge them. Even though the literature seems to have made a great deal of progress in providing an abundance of models and identifying how to estimate its relevant parameters, the question of how derivative instruments can be hedged has largely been overlooked. 
One of the key insights in the work of Black and Scholes (1973) and Merton (1990) was the ability to hedge a vanilla option $V_{1}\left(S, t ; T_{1}, K_{1}\right)$ written on a stock $S_{t}$, that followed a geometric Brownian motion, by forming a portfolio

$$
P(S, t)=V_{1}\left(S, t ; T_{1}, K_{1}\right)-a(S, t) S_{t}
$$

and making it risk-free by setting $a(S, t)=\partial V(S, t) / \partial S$ at every instant in time. It is well known that when $\ln S_{t}$ follows a non-Gaussian process it is not possible for the writer of an option to hedge all the risk by continuously trading in the underlying. Therefore, an alternative approach has been to hedge the option by trading in the underlying and another instrument, say $V_{2}\left(S, t ; T_{2}, K_{2}\right)$, written on the same underlying. This strategy is known as Delta- and Gamma-hedging. The idea behind this approach is to set up a portfolio

$$
P(S, t)=V_{1}\left(S, t ; T_{1}, K_{1}\right)-a(S, t) S_{t}-b(S, t) V_{2}\left(S, t ; T_{2}, K_{2}\right)
$$

and make it Delta- and Gamma-neutral, in other words, choose $a(S, t)$ and $b(S, t)$ so that

$$
\frac{\partial P(S, t)}{\partial S}=0 \quad \text { and } \quad \frac{\partial^{2} P(S, t)}{\partial S^{2}}=0
$$

Although, on average, Delta- and Gamma-hedging generally performs 'better' than Deltahedging, it still leaves the writer of the option considerably exposed to large movements in the underlying stock price. One of the reasons why the traditional Delta- and Gamma-neutral strategy may offer very little protection against large movements in the underlying stock price is because the information upon which the hedging strategy operates is based on 'local' information, ie the first and second derivatives of the portfolio $P(S, t)$ with respect to $S_{t}$. Hence, as long as the stock price does not move by a 'considerable' amount over the next time-step, for which the quantities $a(S, t)$ and $b(S, t)$ are held constant, then the Delta- and Gamma-hedging strategy will offer reasonable protection to the writer of the option. However, if the stock price can jump or exhibit large movements over a small period of time or between rebalances of the 
portfolio, a hedging strategy based on local information such as the Delta and Gamma will perform poorly.

The main contribution of this article is to propose a new dynamic hedging strategy to hedge financial instruments written on securities that follow a non-Gaussian process. We generalise the strategy of Delta- and Gamma-hedging by choosing $a(S, t)$ and $b(S, t)$ based on 'non-local' information, which is obtained by looking at fractional (non-integer) integrals and derivatives of the financial instruments in the portfolio $P(S, t)$. We also show that there is an interesting connection between some of the most popular jump models for equity and fractional calculus. Furthermore we show for the first time that the pricing equations for European-style options, where the underlying follows a wide class of Lévy processes, is given by what we call the Fractional-Black-Scholes (FBS) equation; this is a pricing equation with non-integer derivatives and integral operators, ie fractional operators.

The rest of the paper is structured as follows: Section I introduces the concepts of fractional integrals and fractional derivatives and proposes a novel dynamic hedging strategy, based on fractional derivatives, that can be used to hedge portfolios written on securities that follow nonGaussian processes; Section II describes the family of Lévy processes and looks at specific cases which have become some of the most important models describing the evolution of share prices; Section III tests the dynamic hedging strategies identified in section I when securities follow non-Gaussian processes, including some of the jump processes discussed in section II; Section IV shows that when it is assumed that stock prices follow some of the most popular Lévy-based jump models, for example the CGMY or FMLS (Carr, Geman, Madan, and Yor (2002) and Carr and Wu (2003b)), then the pricing equation satisfied by European-style options written on these stocks satisfy a fractional partial differential equation; and Section V concludes. 


\section{A Dynamic Hedging Strategy for Non-Gaussian Securities}

The principal purpose of this article is to address the question of how financial derivatives can be hedged when the underlying security follows a non-Gaussian process. We will propose, and test, a hedging strategy based on the theory of fractional integration and differentiation. We start by introducing the fundamental concepts of fractional integrals and derivatives as well as a generalisation of Taylor's series that we later employ to develop a new dynamic hedging strategy.

\section{A. Fractional Calculus}

Definition 1 The Riemann-Liouville Fractional Integral. The fractional integral of order $\gamma$ of a function $f(x)$ is given by

$$
{ }_{a} D_{x}^{-\gamma} f(x)=\frac{1}{\Gamma(\gamma)} \int_{a}^{x}(x-y)^{\gamma-1} f(y) d y, \quad \gamma>0
$$

and

$$
{ }_{x} D_{b}^{-\gamma} f(x)=\frac{1}{\Gamma(\gamma)} \int_{x}^{b}(y-x)^{\gamma-1} f(y) d y, \quad \gamma>0
$$

where $\Gamma$ is the gamma function.

For details we refer the reader to Miller and Ross (1993) and Podlubny (1999). ${ }^{1}$

One way to obtain fractional derivatives is to 'view' them as integer derivatives (ie common differentiation) of a fractional integral. 
Definition 2 The Riemann-Liouville Fractional Derivative. If $n$ is the smallest integer larger than the number $\gamma$, then the right and left fractional derivatives of order $\gamma$ of the function $f$ is given by

$$
{ }_{a} D_{x}^{\gamma} f(x)=\frac{1}{\Gamma(n-\gamma)} \frac{d^{n}}{d x^{n}} \int_{a}^{x}(x-y)^{n-\gamma-1} f(y) d y \quad n-1 \leq \gamma<n
$$

and

$$
{ }_{x} D_{b}^{\gamma} f(x)=\frac{(-1)^{n}}{\Gamma(n-\gamma)} \frac{d^{n}}{d x^{n}} \int_{x}^{b}(y-x)^{n-\gamma-1} f(y) d y \quad n-1 \leq \gamma<n
$$

where $n$ is a Natural number and $\Gamma$ is the gamma function.

Note that when $a=-\infty$ and $b=\infty$ the Fourier transforms of the right and left fractional derivatives are given by

$$
\mathcal{F}\left\{{ }_{-\infty} D_{x}^{\gamma} f(x)\right\}=(-i \xi)^{\gamma} \hat{f}(\xi) \quad \text { and } \quad \mathcal{F}\left\{{ }_{x} D_{\infty}^{\gamma} f(x)\right\}=(i \xi)^{\gamma} \hat{f}(\xi)
$$

where

$$
\mathcal{F}\{f(x)\}=\hat{f}(\xi)=\int_{-\infty}^{\infty} e^{i \xi x} f(x) d x \quad \text { and } \quad \xi \in \mathbb{C}
$$

There are also different Taylor's expansions based on fractional operators. We present an example which we later use to develop dynamic hedging strategies.

Proposition 1 Fractional Taylor's Expansion, Samko, Kilbas, and Marichev (1993). Let $\gamma_{0}=0, \gamma_{1}, \ldots, \gamma_{m}$ be an increasing sequence of real numbers such that $0<\gamma_{k}-\gamma_{k-1} \leq 1$, $k=1,2, \ldots, m$. Let $x>0, f(x)$ having all continuous derivatives and introduce the notation

$$
D^{\left\{\gamma_{k}\right\}} f(x)={ }_{0} D_{x}^{-\left(1-\gamma_{k}-\gamma_{k-1}\right)}{ }_{0} D_{x}^{1+\gamma_{k-1}} f(x)
$$


and remark that $D^{\left\{\gamma_{k}\right\}} f(x) \neq{ }_{0} D_{x}^{\gamma_{k}} f(x)$. Then the generalised fractional Taylor's expansion is given by

$$
f(x)=\sum_{k=0}^{m-1} \frac{D^{\left\{\gamma_{k}\right\}} f(0)}{\Gamma\left(1+\gamma_{k}\right)} x^{\gamma_{k}}+\frac{1}{\Gamma\left(1+\gamma_{m}\right)} \int_{0}^{x}(x-y)^{\gamma_{m}-1} D^{\left\{\gamma_{m}\right\}} f(y) d y .
$$

\section{B. Hedging: Delta and Fractional-Gamma neutral strategy}

In practice, the pricing of options is as important as the question of how to hedge them. In the classical Black-Scholes model the hedging of a portfolio $P(S, t)$, consisting of a Europeanstyle derivative $V_{1}\left(S, t ; T_{1}, K_{1}\right)$ expiring at $T_{1}$ and struck at $K_{1}$, and the underlying $S_{t}$, is achieved by continuous Delta-hedging; that is, holding an amount $\partial V_{1}\left(S, t ; T_{1}, K_{1}\right) / \partial S$ of the underlying $S_{t}$ at every instant in time guarantees that the portfolio is risk-free. In practice however, it is impossible to employ a dynamic strategy that requires rebalancing the portfolio at every instant in time; therefore rebalances are done discretely. For example, in the Black-Scholes framework, the less frequently hedges take place the larger the hedging error will be, due mainly to the convexity, known as Gamma, of the value of the option.

Therefore, the classical approach of Delta-hedging a portfolio

$$
P(S, t)=V\left(S, t ; T_{1}, K_{1}\right)-a(S, t) S_{t}
$$

where $a(S, t)$ is the number of shares $S_{t}$ held over the time-step $[t, t+\Delta t)$, requires that $a(S, t)$ is chosen such that the portfolio is Delta-neutral, ie $\partial P(S, t) / \partial S=0$. The hedging error will depend on the size of the higher order terms of the series

$$
\Delta P(S, t)=\frac{\partial P(S, t)}{\partial t} \Delta t+\frac{\partial P(S, t)}{\partial S} \Delta S+\frac{1}{2 !} \frac{\partial^{2} P(S, t)}{\partial S^{2}}(\Delta S)^{2}+\frac{1}{3 !} \frac{\partial^{3} P(S, t)}{\partial S^{3}}(\Delta S)^{3}+\cdots
$$

Hence, if it is not feasible or if it is too expensive to hedge an option as frequently as one would desire, one approach is to use a portfolio as shown in (7), which includes another instrument written on the same underlying, and make this new portfolio both Delta- and Gamma-neutral. 
In other words, form a portfolio consisting of the option to be hedged, say $V_{1}\left(S, t ; T_{1}, K_{1}\right)$, and then choose an amount $a(S, t)$ of the underlying stock and an amount $b(S, t)$ of another instrument, say $V_{2}\left(S, t ; T_{2}, K_{2}\right)$, that is also written on $S_{t}$

$$
P(S, t)=V_{1}\left(S, t ; T_{1}, K_{1}\right)-a(S, t) S_{t}-b(S, t) V_{2}\left(S, t ; T_{2}, K_{2}\right)
$$

so that $\partial P(S, t) / \partial S=0$ and $\partial^{2} P(S, t) / \partial S^{2}=0$.

The rest of this section looks at a new dynamic hedging strategy. We must stress that the hedging strategies we propose below can be applied to hedge any financial instruments and therefore it is not a pre-requisite that the instruments are written on an underlying that follows a particular stochastic process. In Section III, the performance of the hedging strategies will be assessed for different models through the use of simulations; we will look in detail at the hedging of call options when the underlying follows a geometric Lévy-Stable (LS) process, geometric FMLS process (both discussed below in section II) and Merton's Jump-Diffusion (MJD) process. We identify that it is sufficient, using put-call-parity, to set a static hedge in order to hedge vanilla options; however, our objective is to construct a dynamic hedging strategy that will provide insight into the question of how to hedge derivatives written on securities that follow non-Gaussian processes.

Our proposed hedging strategy is based on the generalised Taylor's expansion (6). The idea is the following. Given that most of the processes we are interested in exhibit large ${ }^{2}$ movements or jumps in the underlying stock price, using the classical Delta-hedging strategy will, on average, expose the writer of the option to large hedging errors even if Delta-hedging is performed as often as possible. As mentioned above, one way to proceed is to use a DeltaGamma-neutral strategy. As a generalisation of this approach we propose what we call a Delta- and Fractional-Gamma strategy that instead of making the portfolio Delta-neutral and Gamma-neutral, makes the portfolio Delta-neutral and ${ }_{0} D_{S}^{\gamma} P(S, t)$-neutral with $1<\gamma \leq 2$. 
Therefore the aim is to set up a portfolio $P(S, t)$ to hedge the option $V_{1}\left(S, t ; T_{1}, K_{1}\right)$ by trading in the underlying and another option:

$$
P(S, t)=V_{1}\left(S, t ; T_{1}, K_{1}\right)-a(S, t) S_{t}-b(S, t) V_{2}\left(S, t ; T_{2}, K_{2}\right)
$$

where $V_{i}, i=1,2$, are options written on the underlying $S_{t}$ with expiry $T_{1}<T_{2}$ and struck at $K_{1}$ and $K_{2}$ respectively. The quantities $a(S, t)$ and $b(S, t)$ are the amounts of the underlying and the option $V_{2}\left(S, t ; T_{2}, K_{2}\right)$ that must be held in the hedge portfolio. Therefore, if we look at the change in the value of the portfolio using the generalised fractional Taylor's expansion presented in (6) above, with $\gamma_{0}=0, \gamma_{1}=1$ and $\gamma_{2}=\gamma$ and with $1<\gamma \leq 2$, we obtain

$$
d P(S, t)=\frac{\partial P(S, t)}{\partial S} d S+\frac{{ }_{0} D_{S}^{\gamma} P(S, t)}{\Gamma(1+\gamma)}(d S)^{\gamma}+\cdots
$$

and require

$$
a(S, t)=\frac{\partial V_{1}(S, t)}{\partial S}-\frac{\partial V_{2}(S, t)}{\partial S} b(S, t)
$$

where

$$
b(S, t)=\frac{{ }_{0} D_{S}^{\gamma} V_{1}(S, t)-\partial V_{1}(S, t) / \partial S_{0} D_{S}^{\gamma} S_{t}}{{ }_{0} D_{S}^{\gamma} V_{2}(x, t)-\partial V_{2}(x, t) / \partial S_{0} D_{S}^{\gamma} S_{t}}
$$

such that the portfolio is both Delta- and Fractional-Gamma-neutral, ie

$$
\frac{\partial P(S, t)}{\partial S}=0 \quad \text { and } \quad{ }_{0} D_{S}^{\gamma} P(S, t)=0
$$

For the specific instance where $\gamma=2$, the derivative ${ }_{0} D_{S}^{\gamma} V(S, t)=\partial^{2} V(S, t) / \partial S^{2}$, and this derives the classical Delta- and Gamma-neutral strategy

$$
a(S, t)=\frac{\partial V_{1}(S, t)}{\partial S}-\frac{\partial V_{2}(S, t)}{\partial S} b(S, t) \quad \text { and } \quad b(S, t)=\frac{\partial^{2} V_{1}(S, t) / \partial S^{2}}{\partial^{2} V_{2}(S, t) / \partial S^{2}}
$$

The use of 'non-integer' derivatives to hedge a portfolio is intuitively appealing because the fractional derivative ${ }_{0} D_{S}^{\gamma} P(S, t)$, when $1<\gamma<2$, weighs information about the value of 
the portfolio in the interval $\left[0, S_{t}\right]$ as opposed to only using localised information at the point $S_{t}$. Appendix B depicts fractional derivatives of the value of options written on assets that follow non-Gaussian processes. For example, Figure 10 shows fractional derivatives of an option $V(S, t ; T, K)$ with $T=10$ and $K=100$ where the log-stock price follows an FMLS process of Carr and $\mathrm{Wu}$ (2003b) with $\alpha=1.5$, (see subsection A below where we present the FMLS model). It is clear from the picture that when the traditional measure of Gamma is very close to zero for stock prices below 90 or above 120, the fractional derivatives for values $\gamma=\{2,1.8,1.6,1.4\}$ still have positive values ranging between 0.01 and 0.25 .

Before proceeding it should be noted that it is not necessarily true that performing Deltaand Gamma-hedging is always 'better' than performing Delta-hedging. By inspecting series (8) it may be the case that, even if the second and third terms in the right-hand-side of the equation are zero at the beginning of the time-step $[t, t+\Delta t)$, the higher order terms of the series are of considerable magnitude; this depends on the high order derivatives of the options $V_{1}\left(S, t ; T_{1}, K_{1}\right)$ and $V_{2}\left(S, t ; T_{2}, K_{2}\right)$.

\section{Jump models}

The purpose of this section is twofold. First, since we are interested in testing the proposed fractional hedging strategies described above, we introduce a class of jump models known as Lévy processes and focus on particular members of this class. Second, in section IV, we show another application of fractional calculus in continuous-time finance by showing the connection between fractional pricing equations and the processes presented here.

The use of jump processes to model the dynamics of securities has become a very popular tool over the last decade. Although Brownian motion, the Lévy-Stable (LS) model proposed by Mandelbrot (1997) and jump diffusion models, (see Merton (1990)), belong to the family of Lévy processes, the work of Madan and Seneta (1990) was the first to propose the use of a particular class of Lévy process, known as the Variance Gamma, to model the uncertainty 
underlying security prices. A stochastic process $X_{t}$ is a Lévy process if $X_{0}=0$ and if and only if it has independent and stationary increments. A simple characterisation of Lévy processes is given by the Lévy-Khintchine representation or characteristic function of the process, which we present in Proposition 4 in the Appendix.

One fundamental question that must be answered is what criteria should be employed when choosing a particular Lévy process to model the evolution of a specific underlying. In the case of stock prices this question has been asked, and answered, at different points in time. Arguably the most intuitive and theoretically sound choice of a Lévy model for share prices, is that of Carr, Geman, Madan, and Yor (2002) which is based on the structure of asset returns. Their starting point is to replace Brownian motion, as the driving stochastic component in the formation of prices, with a process that can provide a much richer structure for moments of high order. The authors justify the choice of the Lévy density $w(x)$, which determines the frequency and magnitude of jumps in the process, based on simple, yet very important, characteristics observed in the markets. For example, it seems plausible to expect that the larger the size of the jump in the stock price, the less frequently they occur. Conversely, the smaller the jump size the more frequently they occur. Therefore, by restricting the choice of the density of jumps, and imposing the requirement that exponential moments as well as high order moments exist, a very simple functional form for the Lévy density is arrived at; they labelled the resulting process the CGMY process. Boyarchenko and Levendorskii (2000) also proposed a family of Lévy processes, very similar to the CGMY, called KoBoL or Damped Lévy (DL), which was based on the work of Mantegna and Stanley (2000) and Koponen (1995).

Another interesting choice of Lévy process to model equity prices is in the recent work of Carr and $\mathrm{Wu}$ (2003a). They show that one way to capture the term structure of the implied volatility of S\&P 500 option prices is by assuming that the shocks to the log-stock process follow a maximally skewed LS process; they christened this the Finite Moment Log Stable process (FMLS). 
We now proceed to discuss LS processes for the first time and it will become clear that the other processes (CGMY, DL, KoBoL) can be 'constructed' by introducing an exponential damping in the tails of the LS process.

\section{A. Stock Price Models}

We first look at the LS model introduced by Mandelbrot (1997) in the 1960s. His choice of model was driven by two important considerations. Empirically, Gaussian models do not fit data well due to the fast decay of the tails. Theoretically, if underlying security prices are the cumulative outcome of many small independent events then, by the Generalised Central Limit Theorem, Feller (1966), their cumulative behaviour is characterised by a limiting distribution; namely the LS distribution. One of the most important shortcomings of working with LS processes, with the exception of the Gaussian case, is that variance is infinite and exponential moments, unless the distribution is maximally skewed, do not exist. If $X_{t}$ is an LS process then the behaviour of its jumps is determined by the Lévy density

$$
w_{L S}(x)= \begin{cases}C q|x|^{-1-\alpha} & \text { for } x<0 \\ C p x^{-1-\alpha} & \text { for } x>0\end{cases}
$$

and the natural logarithm of its characteristic function is given in terms of the parameters $\alpha$, $\kappa, \beta$ and $m$ by

$$
\ln \mathbb{E}\left[e^{i \xi X_{t}}\right] \equiv t \Psi(\xi)= \begin{cases}-t \kappa^{\alpha}|\xi|^{\alpha}\{1-i \beta \operatorname{sign}(\xi) \tan (\alpha \pi / 2)\}+i m t \xi & \text { for } \alpha \neq 1 \\ -t \kappa|\xi|\left\{1+\frac{2 i \beta}{\pi} \operatorname{sign}(\xi) \ln |\xi|\right\}+i m t \xi & \text { for } \alpha=1\end{cases}
$$

If the random variable $X$ belongs to an LS distribution with parameters $\alpha, \kappa, \beta, m$, we write $X \sim S_{\alpha}(\kappa, \beta, m)$. The parameter $\alpha$ is known as the stability index or characteristic exponent, $\kappa$ is a scaling parameter, $\beta$ is a skewness parameter and $m$ is a location parameter. We note that if $X$ is an LS random variable with characteristic exponent $0<\alpha \leq 2$, then for the case $0<\alpha \leq 1$ 
the random variable $X$ does not have any integer moments and for the case $1<\alpha<2$ only the first integer moment exists. When $\alpha=2$ the random variable $X$ is Gaussian. Moreover, exponential moments are finite when $X$ is maximally skewed to the left, ie $\beta=-1$.

As mentioned above, another process that has rapidly become a very powerful model for financial securities is the CGMY. This process is a pure jump Lévy process (ie it has no Brownian motion component) with Lévy measure $W(d x)=w_{C G M Y}(x) d x$

$$
w_{C G M Y}(x)= \begin{cases}C \frac{e^{-G|x|}}{|x|^{1+Y}} & \text { for } x<0, \\ C \frac{e^{-M x}}{x^{1+Y}} & \text { for } x>0\end{cases}
$$

and log-characteristic function given by

$$
t \Psi_{C G M Y}(\xi)=t C \Gamma(Y)\left\{(M-i \xi)^{Y}-M^{Y}+(G+i \xi)^{Y}-G^{Y}\right\}
$$

Here $C>0, G \geq 0, M \geq 0$ and $Y<2 .^{3}$ The parameter $C$ may be viewed as a measure of the overall level of activity. The parameters $G$ and $M$ control the exponential decay of the left and right tail respectively. Moreover, when $G=M$, the distribution is symmetric.

Finally, the DL or KoBoL process is also a pure jump Lévy process with Lévy density

$$
w_{D L}(x)= \begin{cases}C q|x|^{-1-\alpha} e^{-\lambda|x|} & \text { for } x<0, \\ C p x^{-1-\alpha} e^{-\lambda x} & \text { for } x>0\end{cases}
$$

whereas in the LS case, $0<\alpha \leq 2, C>0, \lambda \geq 0$ and $p, q \geq 0$ with $p+q=1$. The logcharacteristic function is given by

$$
\begin{aligned}
& t \Psi_{D L}(\xi)=t \kappa^{\alpha}\left\{p(\lambda-i \xi)^{\alpha}+q(\lambda+i \xi)^{\alpha}-\lambda^{\alpha}\right\}+i m t \xi \\
& t \Psi_{D L}(\xi)=t \kappa^{\alpha}\left\{p(\lambda-i \xi)^{\alpha}+q(\lambda+i \xi)^{\alpha}-\lambda^{\alpha}-i \xi \alpha \lambda^{\alpha-1}(q-p)\right\}+i m t \xi
\end{aligned}
$$

for $0<\alpha<1$ and for $1<\alpha \leq 2$ respectively. 
When $X$ belongs to a DL distribution with parameters $\alpha, \kappa, p, q, m$ and $\lambda$ we write $X \sim D L_{\alpha}(\kappa, p, q, m, \lambda)$ and the parameters have a similar interpretation as in the LS process. Note that $\lambda$ introduces an exponential damping in the tails of the distribution and the DL and LS are the same when $\lambda=0 .{ }^{4}$

Before testing the proposed fractional hedging strategies we must also discuss the connection between the risk-neutral and statistical dynamics of the stock price. The pricing of financial instruments, where the underlying security is modelled via exponential Lévy processes, is not as straightforward as that based on Brownian motion. Markets are not complete when there are jumps in the underlying stock price and therefore there is no unique martingale measure under which prices are calculated. It is the market who 'chooses' the corresponding risk-neutral EMM. Moreover, in theory, hedging strategies could be constructed where every possible jump in the underlying is hedged with another financial instrument. However, this seems impossible in practice since a continuum of options would be required for such a strategy to work.

In the Lévy process literature it is generally assumed that the underlying security follows both a Lévy process under the physical and risk-neutral measures and different EMMs have been proposed to link these two measures. One simple method to derive an EMM is to assume that the process under both the physical and statistical measures has the same shape but a different location. Another method is to use the Esscher transform, see Schoutens (2003), Cont and Tankov (2004). ${ }^{5}$

In the case of the CGMY process, Carr, Geman, Madan, and Yor (2002) assume that log-stock prices follow a CGMY process under the statistical and physical measures where the risk-neutral parameters of the stock process, say $\tilde{C}, \tilde{G}, \tilde{M}$ and $\tilde{Y}$, may differ from their statistical counterparts $C, G, M$ and $Y$.

Finally, McCulloch (2003) showed that, if it is assumed that under the physical measure asset prices follow a geometric LS process (with no restriction on the skewness of the distribution of the LS shocks), then the pricing of derivatives under the risk-neutral measure can be 
performed by assuming that the logarithm of the underlying follows a combination of two independent processes: a maximally negatively skewed process (ie the FMLS) and a maximally skewed to the right DL process (ie $p=1$ in (18)).

\section{Simulations}

In this section we test how our Delta- and Fractional-Gamma-neutral strategy performs. We have assumed that there are no transaction costs and that the markets are liquid. For illustrative purposes we will compare the profit and loss $(\mathrm{P} \& \mathrm{~L})$ obtained from hedging a portfolio using the fractional strategy proposed above, with the results given by performing the more common Delta- and Gamma-neutral strategy. For completeness, we also show what happens when only Delta-hedging is employed and in Appendix $\mathrm{C}$ we show how to evaluate the fractional derivatives used in the hedging strategies. We look in detail at the FMLS process, the LS process, and the MJD process.

\section{A. Hedging in the FMLS model}

Here we illustrate how the Fractional-hedging strategy performs when compared to simple Delta-hedging and to the more common Delta- and Gamma-neutral hedging strategies, when the log-stock process follows an FMLS process. In this model the statistical dynamics of the stock price are given by

$$
d\left(\ln S_{t}\right)=\mu d t+\sigma d L_{t}^{F M L S}
$$

and under the risk-neutral measure it follows

$$
d\left(\ln S_{t}\right)=\left(r+\sigma^{\alpha} \sec (\alpha \pi / 2)\right) d t+\sigma d L_{t}^{F M L S}
$$

where $\mu>0, d L_{t}^{F M L S} \sim S_{\alpha}\left(d t^{1 / \alpha},-1,0\right)$ is a maximally skewed LS motion and $1<\alpha \leq 2$. 
In order to test the proposed dynamic strategy we must simulate price paths for the maximally skewed LS motion. The shocks to an LS motion are given by $\Delta t^{1 / \alpha} \phi$ where $\phi \sim S_{\alpha}(1, \beta, 0)$ and $\Delta t$ is the time-step. Skewed LS random variables can be constructed by combining symmetric LS; Proposition 6 in Appendix D shows how symmetric LS random variables can be generated.

Figure 1 shows a histogram of the P\&L function of a portfolio that has been Delta-hedged daily for a European call option expiring in one month, ie $T_{1}=20$ working days. The logstock price follows an FMLS process with $\mu=.05, \sigma=0.20, \beta=-1$ and $\alpha=1.5$. We have assumed that $r=0, S_{0}=100, K_{1}=100$, that the stock pays no dividends, and we have performed $N=10,000$ simulations. As expected, performing Delta-hedging is not enough to hedge the frequent and often sizeable jumps in the underlying. On average, the P\&L of the Delta-hedged portfolio is $£-0.06$ but with a standard deviation of 5.01 and values ranging from $\min =£-94.75$ to $\max =£ 2.43$. Although it is impossible to hedge all of the jumps in the underlying, one possibility is to hedge the portfolio, using a second option written on the same underlying, by making it Delta- and Gamma-neutral. The second option used in the hedge portfolio had an expiry date of $T_{2}=25$ working days and a strike of $K_{2}=100$. Figure 2 shows the results for this strategy. As expected, since we are using two instruments in the hedging strategy, the results are considerably better than those resulting from simple Delta-hedging. On average the P\&L function of the Delta- and Gamma-neutral portfolio is $£-0.007$ with a standard deviation of 1.58 and values ranging between $\min =£-57.69$ and $\max =£ 46.10$. Finally, Figure 3 shows the results from employing our proposed Delta- and Fractional-Gamma-neutral strategy with $\gamma=1.5$. The improvement over the traditional Deltaand Gamma-neutral strategy is substantial. The fractional strategy considerably reduces the exposure to large movements in the underlying. The mean of the P\&L function is $£-0.0004$ with a standard deviation of 0.42 and values ranging from $\min =£-8.03$ to $\max =£ 18.16$. Table I summarises these results and also shows the results from Delta- and Fractional-Gamma hedging for a range of values between 1.1 and 1.7 for $\gamma$. It is important to note that the fractional strategy that delivered the smallest standard deviation of the P\&L was $\gamma=1.5$ and the 


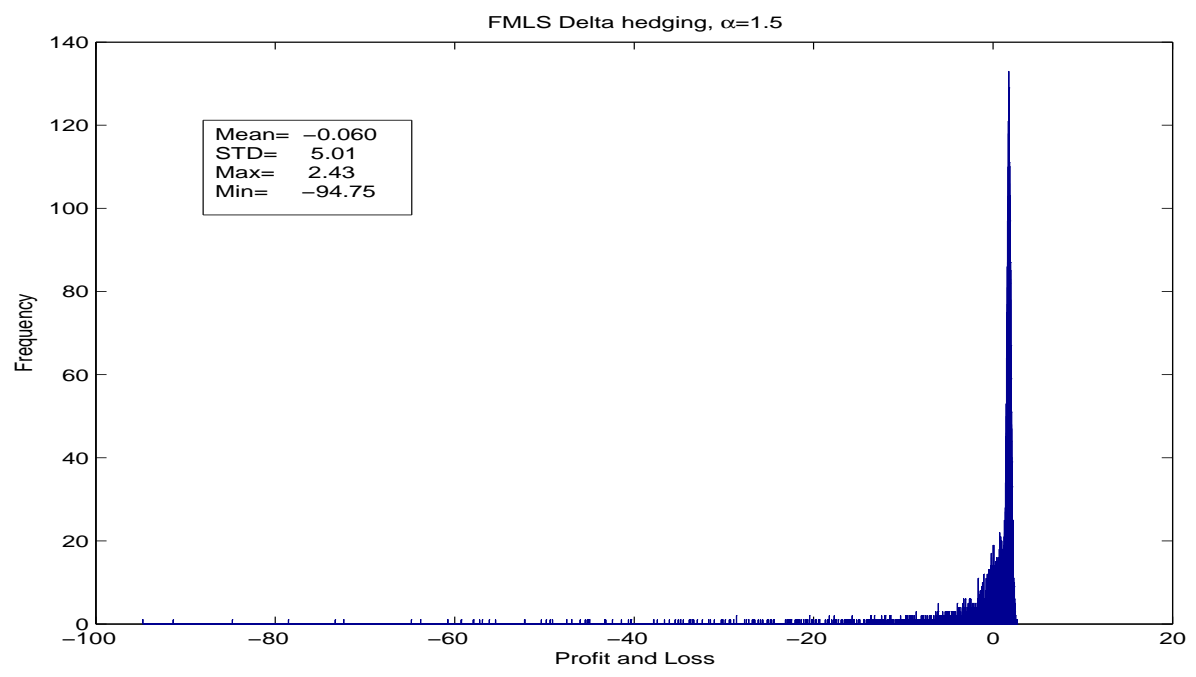

Figure 1. Daily Delta-Hedging. P\&L resulting from daily Delta-hedging under the assumption that the stock price follows an FMLS process.

one that delivered the highest lower bound for the P\&L was the one performed using $\gamma=1.6$. Moreover, we repeat the simulations but vary the strike price of the second option. Table II summarises the results from using $K_{2}=95$ and Table III from using $K_{2}=105$. In both cases the fractional strategies deliver better results than Delta- and Gamma-hedging.

\begin{tabular}{|c|ccccccccc|}
\hline & \multicolumn{7}{|c|}{ FMLS, $\alpha=1.5, S_{0}=100, K_{1}=100, K_{2}=100, T_{1}=20, T_{2}=25$} \\
\hline & $\gamma=1.1$ & $\gamma=1.2$ & $\gamma=1.3$ & $\gamma=1.4$ & $\gamma=1.5$ & $\gamma=1.6$ & $\gamma=1.7$ & $\gamma=2$ & Delta \\
\hline Mean & -0.0029 & -0.0028 & -0.0025 & -0.0016 & -0.0004 & 0.0034 & 0.0063 & -0.0070 & -0.0604 \\
STD & 0.5191 & 0.5113 & 0.4937 & 0.4606 & 0.4288 & 0.4853 & 0.6741 & 1.5894 & 5.0198 \\
Max & 21.02 & 20.77 & 20.30 & 19.57 & 18.16 & 20.70 & 25.40 & 46.10 & 2.43 \\
Min & -12.77 & -12.39 & -11.55 & -9.99 & -8.03 & -7.78 & -12.81 & -57.69 & -94.75 \\
\hline
\end{tabular}

Table I

P\&L statistics from $N=10,000$ simulations. We show Delta- and Fractional-Gamma-neutral strategies for various $\gamma$ 's and the last two columns show the Delta-Gamma-neutral strategy (ie $\gamma=2$ ) and Delta-neutral strategy respectively. 


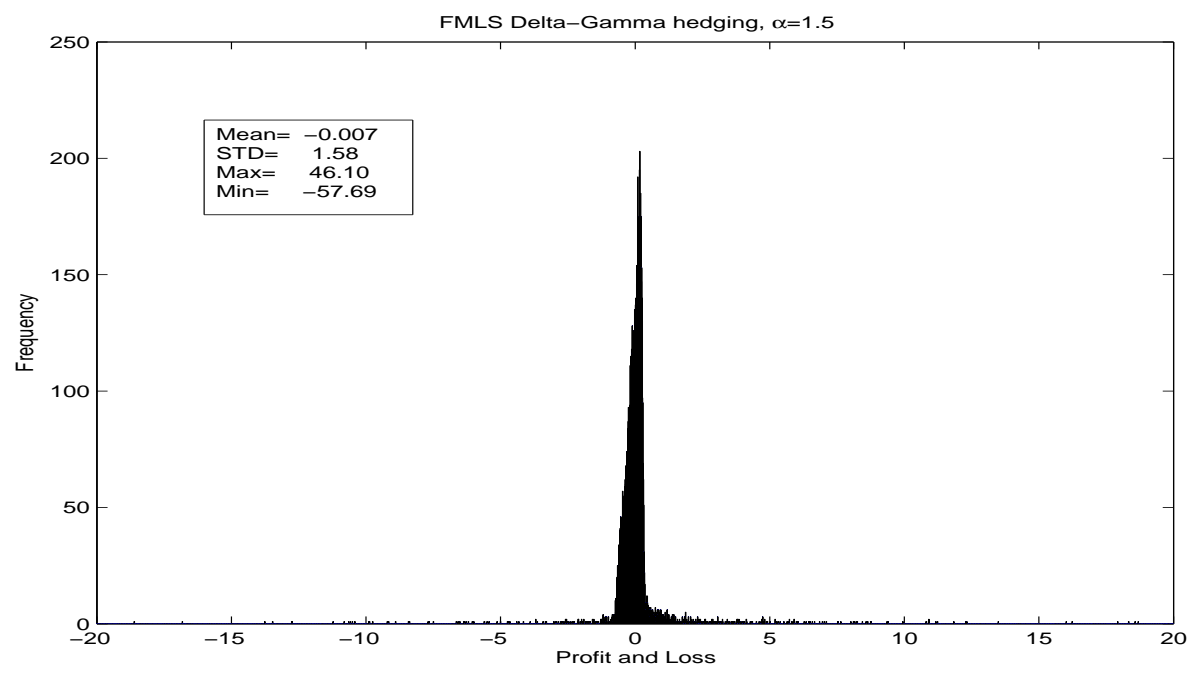

Figure 2. Daily Delta- and Gamma-Hedging. P\&L resulting from employing a Delta- and Gamma-neutral strategy when the underlying follows an FMLS process.

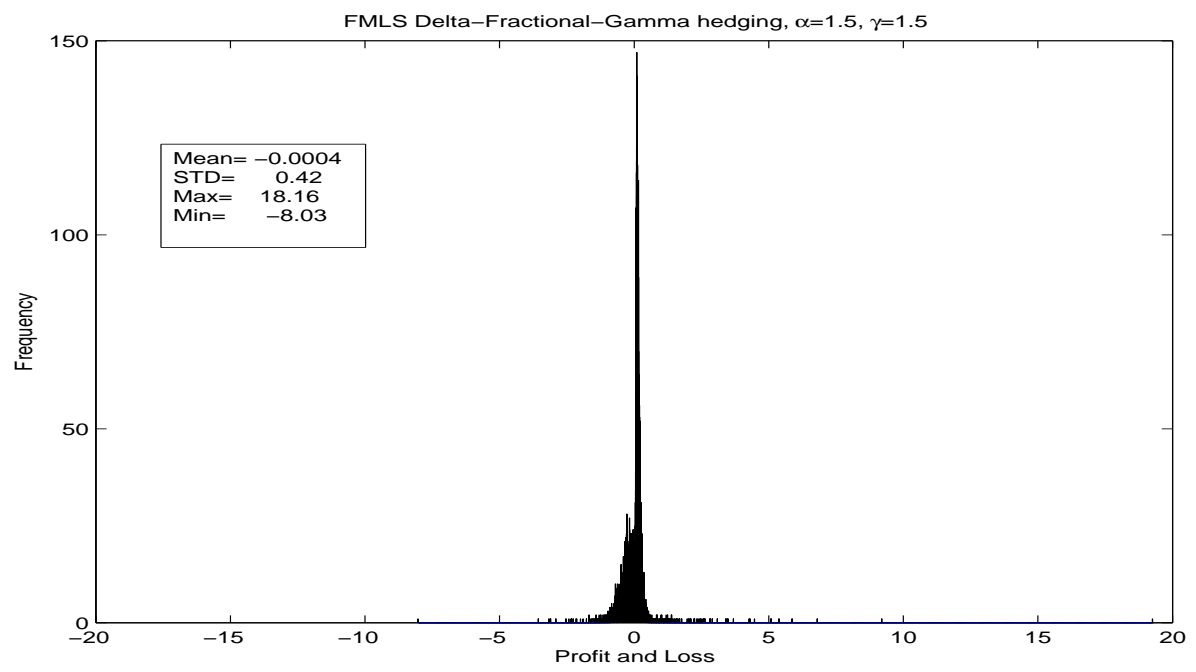

Figure 3. Daily Delta- and Fractional-Gamma-Hedging. P\&L resulting from employing a Delta- and Fractional-Gamma strategy when the underlying follows an FMLS process. 


\begin{tabular}{|c|ccccccc|cc|}
\hline & \multicolumn{7}{|c|}{ FMLS, $\alpha=1.5, S_{0}=100, K_{1}=100, K_{2}=95, T_{1}=20, T_{2}=25$} \\
\hline & $\gamma=1.1$ & $\gamma=1.2$ & $\gamma=1.3$ & $\gamma=1.4$ & $\gamma=1.5$ & $\gamma=1.6$ & $\gamma=1.7$ & $\gamma=2$ & Delta \\
\hline Mean & -0.0284 & -0.0285 & -0.0288 & -0.0295 & -0.0312 & -0.0339 & -0.0374 & -0.1540 & 0.0946 \\
STD & 1.6325 & 1.6281 & 1.6187 & 1.6011 & 1.5774 & 1.5729 & 1.6291 & 5.7067 & 4.0185 \\
Max & 49.54 & 49.24 & 48.49 & 46.69 & 42.82 & 37.35 & 41.47 & 197.87 & 2.46 \\
Min & -5.29 & -5.24 & -5.23 & -5.22 & -5.22 & -5.27 & -6.18 & -30.57 & -81.00 \\
\hline
\end{tabular}

Table II

P\&L statistics from $N=10,000$ simulations. We show Delta- and Fractional-Gamma-neutral strategies for various $\gamma$ 's and the last two columns show the Delta-Gamma-neutral strategy (ie $\gamma=2$ ) and Delta-neutral strategy respectively.

\begin{tabular}{|c|ccccccccc|}
\hline & \multicolumn{7}{|c|}{ FMLS, $\alpha=1.5, S_{0}=100, K_{1}=100, K_{2}=105, T_{1}=20, T_{2}=25$} \\
\hline & $\gamma=1.1$ & $\gamma=1.2$ & $\gamma=1.3$ & $\gamma=1.4$ & $\gamma=1.5$ & $\gamma=1.6$ & $\gamma=1.7$ & $\gamma=2$ & Delta \\
\hline Mean & 0.0017 & 0.0017 & 0.0017 & 0.0016 & 0.0015 & 0.0011 & 0.0004 & -0.0043 & 0.0087 \\
STD & 2.3193 & 2.3166 & 2.3097 & 2.2936 & 2.2614 & 2.2136 & 2.1705 & 2.4742 & 4.6324 \\
Max & 4.07 & 4.07 & 4.06 & 4.03 & 3.96 & 3.79 & 3.97 & 1.64 & 2.37 \\
Min & -45.56 & -45.52 & -45.39 & -45.07 & -44.36 & -43.21 & -42.04 & -56.82 & -89.84 \\
\hline
\end{tabular}

Table III

P\&L statistics from $N=10,000$ simulations in the $\mathbf{L S}$ model. We show Delta-Fractional-Gamma-neutral strategies for various $\gamma$ 's and the last two columns show the Delta- and Gamma-neutral strategy (ie $\gamma=2$ ) and Delta-neutral strategy respectively.

\section{B. Hedging in the LS model}

Here we assume that under the physical measure the price process follows a geometric LS process

$$
d\left(\ln S_{t}\right)=\mu d t+\sigma d L_{t}^{L S},
$$

where $d L_{t}^{L S} \sim S_{\alpha}\left(d t^{1 / \alpha}, \beta, 0\right)$ with $0 \leq \alpha \leq 2,-1 \leq \beta \leq 1, \mu>0$ and $\sigma>0$. Under the risk-neutral measure, see (D9) in the appendix, it follows that

$$
d\left(\ln S_{t}\right)=\left(r-\beta \sigma^{\alpha} \sec (\alpha \pi / 2)\right) d t+\sigma d \tilde{L}_{t}^{L S}+\sigma d \tilde{L}_{t}^{D L}
$$


where $d \tilde{L}_{t}^{L S}$ and $d \tilde{L}_{t}^{D L}$ are independent and as stated in (D10). This is not only an interesting case from a financial point of view but also one that can be used to stress-test hedging strategies given the heavy tails of the process. As mentioned earlier, the shocks to the stock dynamics shown in (19) above, have infinite variance and exponential moments do not exist.

We proceed as above and compare the results of hedging a European call option, (where $S_{0}=100, K_{1}=100$ and $T_{1}=20$ ) with a second option (where $K_{2}=100$ and $T_{2}=25$ ), using the Delta- and Fractional-Gamma-neutral strategy, with the more familiar Delta- and Gammaneutral strategy. For illustrative purposes we simulate stock prices using Proposition 6 (in Appendix D) when $\alpha=1.7, \beta=-0.5, \mu=0.05$ and $\sigma=0.20$. Moreover, for simplicity, we assume that the risk-free rate $r=0$ and that the stock pays no dividends.

Figures 4, 5 and 6 show histograms of the P\&L and Table IV summarises the results of the simulations using a range of fractional derivatives. We highlight that in this case we cannot show the standard deviation of the P\&L since, under the physical measure, exponential moments of the log-stock price do not exist. Note that when the traditional Delta- Gammaneutral strategy is employed, assuming $K_{2}=100$, the resulting $\mathrm{P} \& \mathrm{~L}$ is within the interval $[-101.46,630.04]$ which contrasts sharply with the range $[-5.45,7.70]$, obtained when fractional strategies are used with $\gamma=1.4$. Moreover, Table $\mathrm{V}$ shows the results from another set of simulations assuming that the strike of the second option is $K_{2}=105$. In this case the DeltaGamma-neutral strategy delivers P\&L results within the interval $[-41.66,452.15]$ whereas for all the fractional strategies the resulting P\&L lie between $[-39,81]{ }^{6}$ 


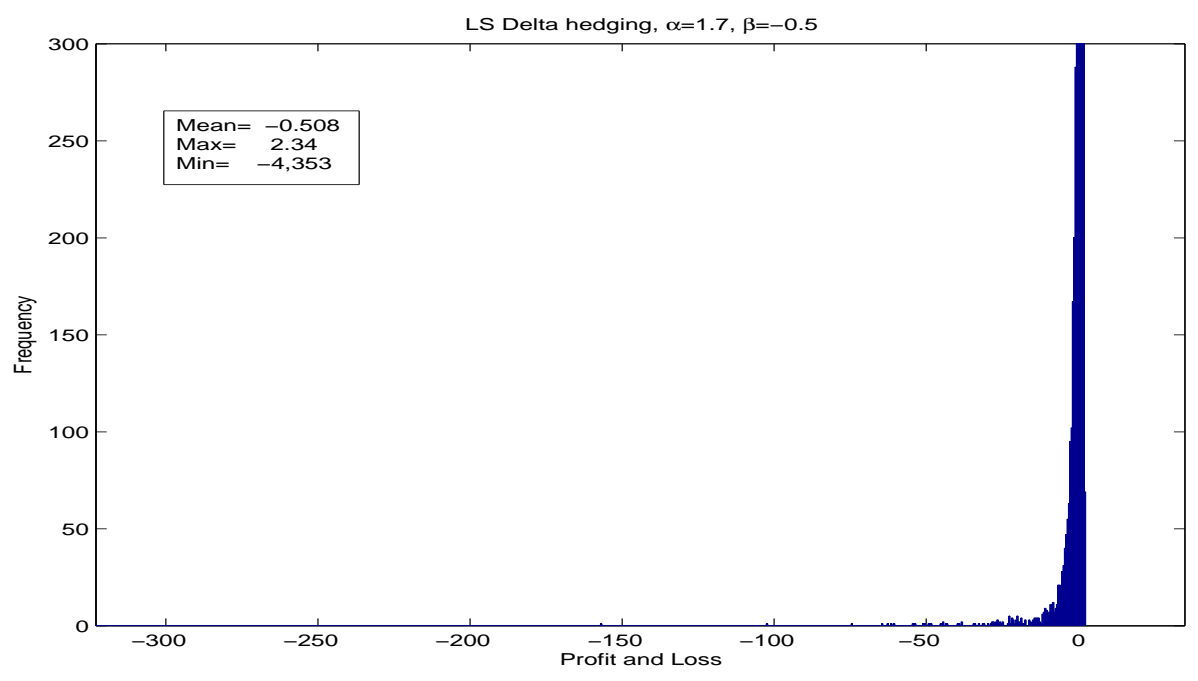

Figure 4. Daily Delta-Hedging. P\&L resulting from using the Black-Scholes Delta-hedging strategy.

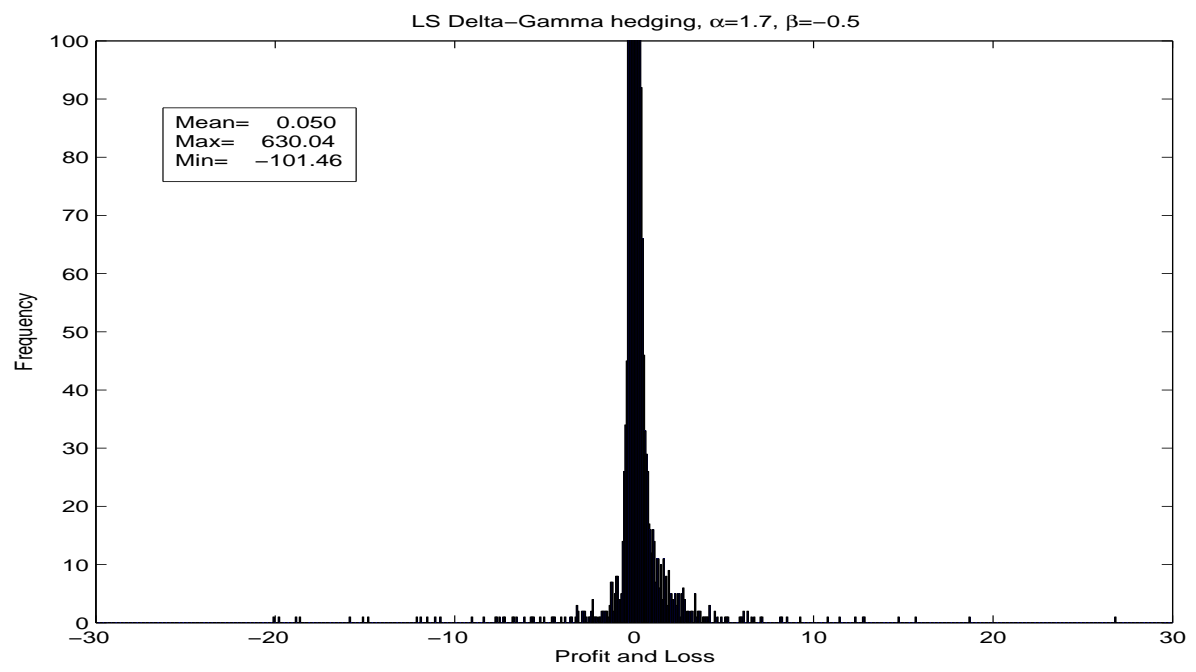

Figure 5. Daily Delta- and Gamma-Hedging. P\&L resulting from using a Delta- and Gamma-neutral strategy. 


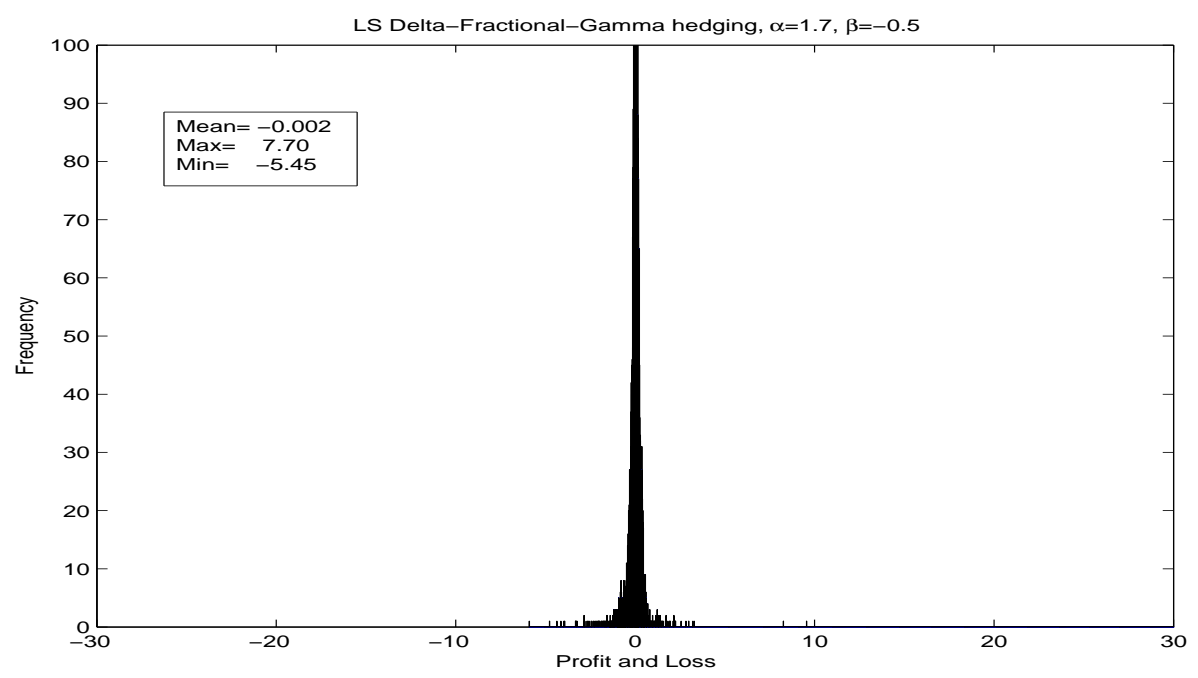

Figure 6. Daily Delta- and Fractional-Gamma-Hedging. P\&L resulting from using a Deltaand Fractional-Gamma strategy with $\gamma=1.4$.

\section{Hedging in the MJD model}

Here we show how Delta- and Fractional-Gamma-hedging compares to Delta- and Gammahedging when the underlying security follows a jump diffusion model as proposed by Merton (1990). This model proposes that under the physical measure, $S_{t}$ follows

$$
\frac{d S_{t}}{S_{t^{-}}}=\mu d t+\sigma d W_{t}+(J-1) d q_{t}
$$

where $\mu$ is a constant, $\sigma \geq 0, d W_{t}$ is the increment of a standard Wiener process, $q_{t}$ is a Poisson process with intensity parameter $\lambda t$ and $J_{i}$ is a sequence of i.i.d. random variables such that $\ln J \sim N\left(\mu_{J}, \sigma_{J}^{2}\right)$ and $W_{t}, q_{t}$ and $J_{i}$ 's are independent.

We assume that under the risk-neutral measure the stock price follows

$$
\frac{d S_{t}}{S_{t^{-}}}=\left(r-\frac{1}{2} \sigma^{2}-\lambda\left(\mathbb{E}_{J}[J]-1\right)\right) d t+\sigma d \tilde{W}_{t}+(J-1) d q_{t}
$$

where $r$ is the risk-free rate and $d \tilde{W}_{t}$ is the increment of Brownian motion. 


\begin{tabular}{|c|cccccccccc|}
\hline & \multicolumn{7}{|c|}{ LS, $\alpha=1.7, \beta=-0.5, S_{0}=100, K_{1}=100, K_{2}=100, T_{1}=20, T_{2}=25$} \\
\hline & $\gamma=1.1$ & $\gamma=1.2$ & $\gamma=1.3$ & $\gamma=1.4$ & $\gamma=1.5$ & $\gamma=1.6$ & $\gamma=1.7$ & $\gamma=2$ & Delta \\
\hline Mean & -0.0073 & -0.0069 & -0.0059 & -0.0027 & 0.0050 & 0.019 & 0.039 & 0.055 & -0.508 \\
Max & 7.19 & 7.25 & 7.40 & 7.70 & 63.70 & 190.00 & 362.72 & 630.04 & 2.34 \\
Min & -37.18 & -34.51 & -25.77 & -5.45 & -5.89 & -8.62 & -12.30 & -101.46 & $-4,353$ \\
\hline
\end{tabular}

Table IV

P\&L statistics from $N=10,000$ simulations in the $\mathbf{L S}$ model. We show Delta-Fractional-Gamma-neutral strategies for various $\gamma$ 's and the last two columns show the Delta- and Gamma-neutral strategy (ie $\gamma=2$ ) and Delta-neutral strategy respectively.

\begin{tabular}{|c|ccccccccc|}
\hline & \multicolumn{7}{|c|}{ LS, $\alpha=1.7, \beta=-0.5, S_{0}=100, K_{1}=100, K_{2}=105, T_{1}=20, T_{2}=25$} \\
\hline & $\gamma=1.1$ & $\gamma=1.2$ & $\gamma=1.3$ & $\gamma=1.4$ & $\gamma=1.5$ & $\gamma=1.6$ & $\gamma=1.7$ & $\gamma=2$ & Delta \\
\hline Mean & 0.0268 & 0.0269 & 0.0270 & 0.0274 & 0.0285 & 0.0306 & 0.0337 & 0.1069 & -0.0449 \\
Max & 74.47 & 74.67 & 75.12 & 76.04 & 77.62 & 79.47 & 80.31 & 452.15 & 2.53 \\
Min & -38.46 & -38.37 & -38.17 & -37.73 & -36.93 & -35.82 & -34.92 & -41.66 & -199.12 \\
\hline
\end{tabular}

Table V

P\&L statistics from $N=10,000$ simulations in the $\mathbf{L S}$ model. We show Delta-Fractional-Gamma-neutral strategies for various $\gamma$ 's and the last two columns show the Delta- and Gamma-neutral strategy (ie $\gamma=2$ ) and Delta-neutral strategy respectively.

Table VI shows results from $N=10,000$ simulations for the MJD model with parameters $\mu=.05, \sigma=0.2, \sigma_{J}=0.2, \mu_{J}=0$ and assuming that the stock price jumps on average $\lambda=$ 5 times per year. As above, we have assumed that $r=0, T_{1}=20, T_{2}=25, K_{1}=K_{2}=$ 100 and $S_{0}=100$. It can be appreciated from the results that the Delta- Fractional-Gamma strategy with $1.1 \leq \gamma \leq 1.7$ delivers considerably better results than the Delta- Gamma-neutral strategy. Figures 7 and 8 show the histograms for the P\&L resulting from the simulations for Delta hedging and Delta- and Gamma hedging. Figure 9 shows the histogram for Delta- and Fractional-Gamma hedging when $\gamma=1$.1. This value delivered the lowest volatility, which is approximately $30 \%$ of the volatility of the P\&L resulting from the classical Delta- and Gamma 


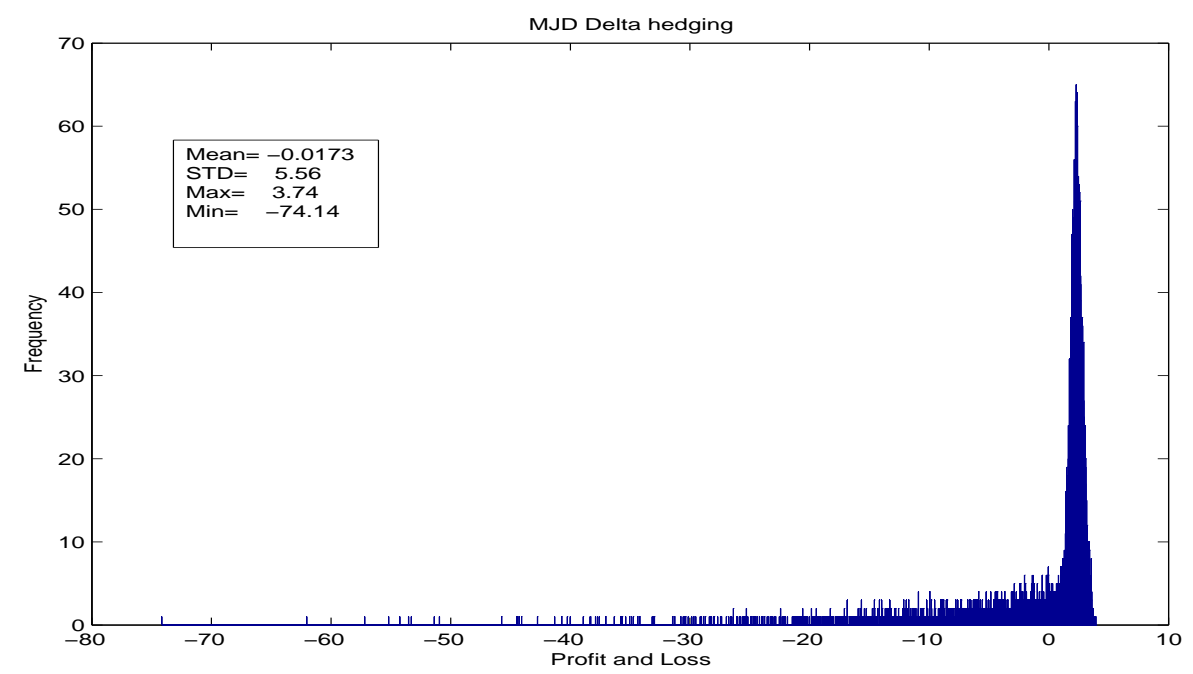

Figure 7. Daily Delta-Hedging. P\&L resulting from using the Black-Scholes Delta-hedging strategy.

hedging strategy. Moreover, Tables VII and VIII show simulations for the cases where $K_{2}=95$ and $K_{2}=105$ respectively.

\begin{tabular}{|l|ccccccccc|}
\hline & \multicolumn{7}{|c|}{ MJD, $S_{0}=100, K_{1}=100, K_{2}=100, T_{1}=20, T_{2}=25, \lambda=5$} \\
\hline & $\gamma=1.1$ & $\gamma=1.2$ & $\gamma=1.3$ & $\gamma=1.4$ & $\gamma=1.5$ & $\gamma=1.6$ & $\gamma=1.7$ & $\gamma=2$ & Delta \\
\hline Mean & 0.0005 & 0.0004 & 0.0001 & -0.0008 & -0.0033 & -0.0086 & -0.0168 & -0.0521 & -0.0173 \\
STD & 0.7453 & 0.7465 & 0.7492 & 0.7582 & 0.7975 & 0.9361 & 1.2306 & 2.4194 & 5.5602 \\
Max & 10.07 & 10.23 & 10.66 & 11.66 & 13.67 & 16.48 & 21.89 & 41.90 & 3.74 \\
Min & -9.87 & -10.08 & -10.60 & -11.86 & -14.83 & -20.99 & -31.53 & -68.28 & -74.14 \\
\hline
\end{tabular}

Table VI

P\&L statistics from $N=10,000$ simulations in the $\mathbf{L S}$ model. We show Delta-Fractional-Gamma-neutral strategies for various $\gamma$ 's and the last two columns show the Delta- and Gamma-neutral strategy (ie $\gamma=2$ ) and Delta-neutral strategy respectively. 


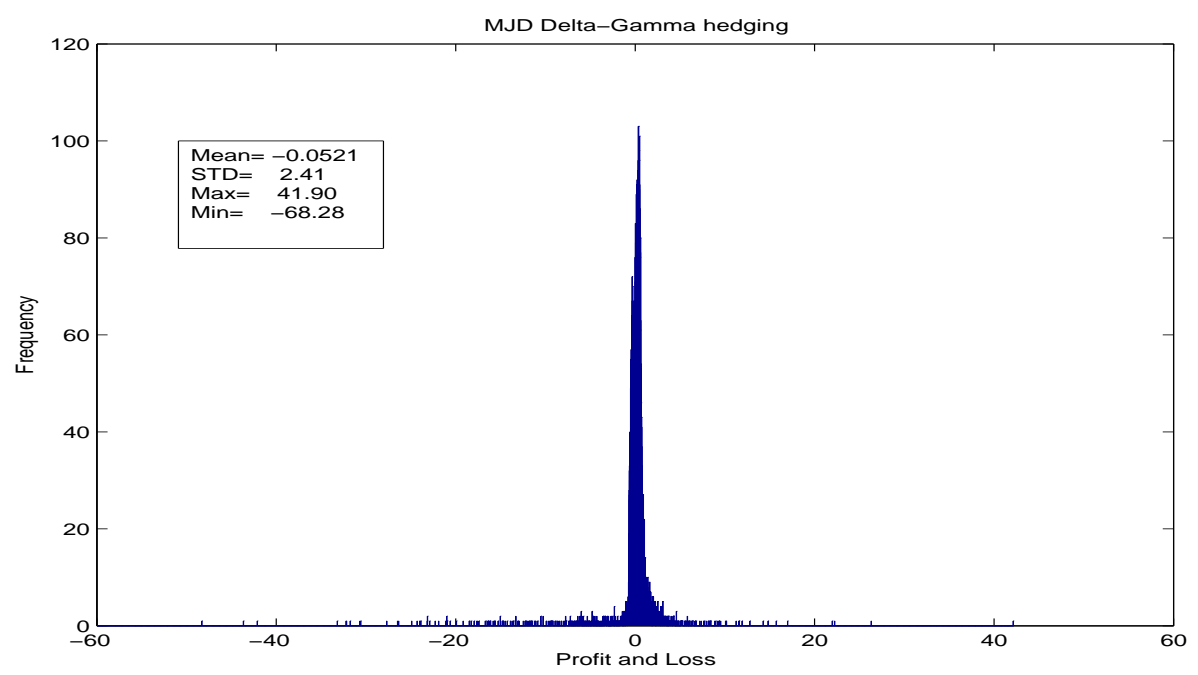

Figure 8. Daily Delta- and Gamma-Hedging. P\&L resulting from using a Delta- and Gamma-neutral strategy.

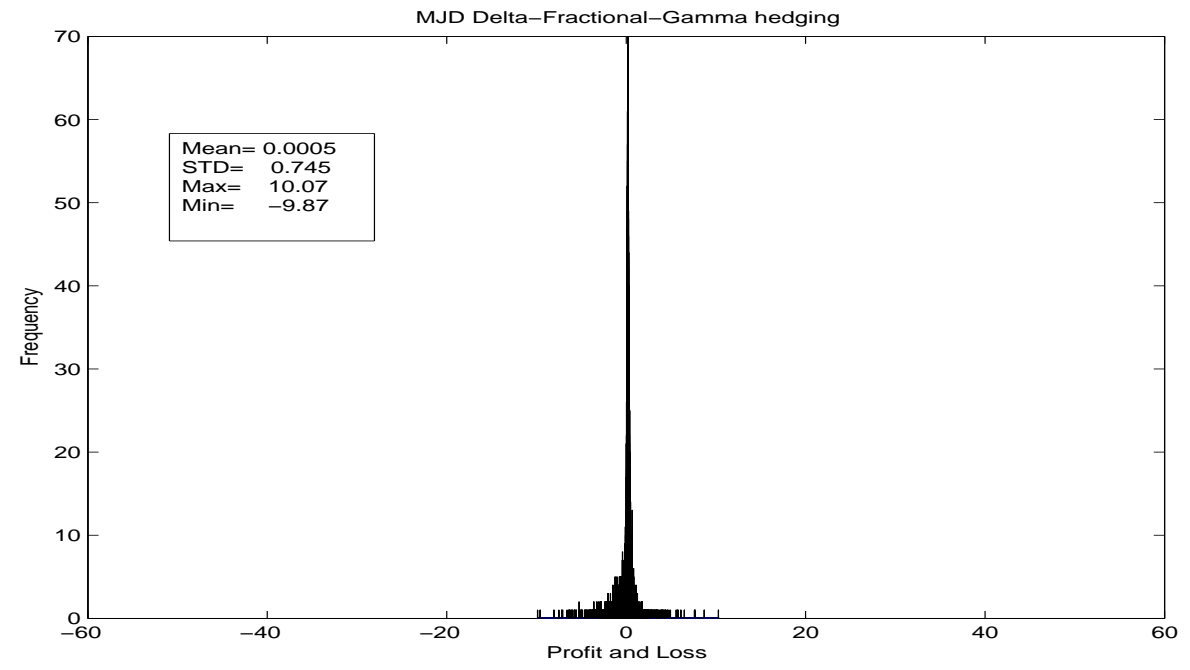

Figure 9. Daily Delta- and Fractional-Gamma-Hedging. P\&L resulting from using a Deltaand Fractional-Gamma strategy with $\gamma=1.1$. 


\begin{tabular}{|c|ccccccc|cc|}
\hline & \multicolumn{7}{|c|}{ MJD, $S_{0}=100, K_{1}=100, K_{2}=95, T_{1}=20, T_{2}=25, \lambda=5$} \\
\hline & $\gamma=1.1$ & $\gamma=1.2$ & $\gamma=1.3$ & $\gamma=1.4$ & $\gamma=1.5$ & $\gamma=1.6$ & $\gamma=1.7$ & $\gamma=2$ & Delta \\
\hline Mean & -0.0044 & -0.0043 & -0.0043 & -0.0044 & -0.0048 & -0.0060 & -0.0085 & -0.0282 & -0.0418 \\
STD & 2.66 & 2.66 & 2.66 & 2.65 & 2.66 & 2.69 & 2.78 & 8.42 & 5.44 \\
Max & 23.94 & 23.77 & 23.36 & 22.38 & 20.48 & 19.26 & 22.28 & 263.53 & 3.80 \\
Min & -38.45 & -38.51 & -38.65 & -38.96 & -39.57 & -40.47 & -41.06 & -51.11 & -55.57 \\
\hline
\end{tabular}

Table VII

P\&L statistics from $N=10,000$ simulations in the $\mathbf{L S}$ model. We show Delta-Fractional-Gamma-neutral strategies for various $\gamma$ 's and the last two columns show the Delta- and Gamma-neutral strategy $(i e=2)$ and Delta-neutral strategy respectively.

\begin{tabular}{|c|ccccccc|cc|}
\hline & \multicolumn{7}{|c|}{ MJD, $S_{0}=100, K_{1}=100, K_{2}=105, T_{1}=20, T_{2}=25, \lambda=5$} \\
\hline & $\gamma=1.1$ & $\gamma=1.2$ & $\gamma=1.3$ & $\gamma=1.4$ & $\gamma=1.5$ & $\gamma=1.6$ & $\gamma=1.7$ & $\gamma=2$ & Delta \\
\hline Mean & -0.0483 & -0.0485 & -0.0489 & -0.0499 & -0.0519 & -0.0547 & -0.0573 & -0.0899 & 0.0302 \\
STD & 2.49 & 2.49 & 2.50 & 2.54 & 2.62 & 2.77 & 3.02 & 12.96 & 5.35 \\
Max & 38.71 & 38.86 & 39.20 & 39.85 & 40.88 & 41.87 & 48.07 & 321.89 & 3.98 \\
Min & -23.36 & -23.32 & -23.26 & -23.21 & -23.30 & -23.86 & -38.56 & -58.27 & -67.43 \\
\hline
\end{tabular}

\section{Table VIII}

P\&L statistics from $N=10,000$ simulations in the $\mathbf{L S}$ model. We show Delta-Fractional-Gamma-neutral strategies for various $\gamma$ 's and the last two columns show the Delta- and Gamma-neutral strategy (ie $\gamma=2$ ) and Delta-neutral strategy respectively.

\section{Other Applications: Fractional Black-Scholes equations}

The pricing of European-style options written on assets that follow non-Gaussian processes, such as Lévy processes, has become a very straightforward task when transform methods are used, Carr and Madan (1999) and Lewis (2001). On the other hand, although progress has been made with regards to the pricing of other types of options, such as American and exotic, there is still scope to develop better and more accurate methods. 
Although until now the theory of fractional calculus had not been applied to the field of finance, there is a wealth of literature, and associated findings, from the theory of fractional differential equations that may prove useful in helping to solve current problems in continuous-time finance. Therefore in this section we show another way in which financial instruments are closely related to fractional calculus. We show that if the risk-neutral dynamics of the log-stock process follow a Lévy process such as the specific cases discussed above in section II, then the corresponding pricing equation satisfied by instruments written on these assets satisfies a FBS, which is a pricing equation with fractional derivatives or fractional integrals. Below, we use the following proposition to show the connection between these families of Lévy processes and their corresponding FBS equations. The proposition shows that the (Fourier transformed) value of a European-style option, where the underlying follows a Lévy process, satisfies an ordinary differential equation (ODE).

Proposition 2 The Pricing ODE. Let $x_{t}=\ln S_{t}$ follow, under the risk-neutral measure,

$$
d x_{t}=\mu d t+\sigma d L_{t}
$$

where $S_{t}$ is the underlying stock price, $\mu$ and $\sigma$ are constants and $d L_{t}$ is the increment of a Lévy process with log-characteristic function $\Psi(\xi)$. Moreover, we let

$$
\hat{V}(\xi, t)=\int_{-\infty}^{\infty} e^{i \xi x} V(x, t) d x, \quad \text { with } \quad \xi \in \mathbb{C}
$$

denote the Fourier transform of the value of a European-style option with final payoff $\Pi\left(x_{T}, T\right)$. Then $\hat{V}(\xi, t)$ satisfies the $O D E$

$$
\frac{\partial \hat{V}(\xi, t)}{\partial t}=[r+i \xi \mu-\Psi(-\xi)] \hat{V}(\xi, t)
$$

with boundary condition $\hat{V}(\xi, T)=\hat{\Pi}(\xi, T)$.

For a proof see Appendix D. 
Here we show that if the risk-neutral dynamics of the stock price follow a LS, CGMY, DL or KoBoL process then the pricing equation satisfied by European-style derivatives contains fractional derivatives and integrals.

Proposition 3 The Lévy-Stable FBS equation. Let the log-price $x_{t}=\ln S_{t}$ process follow, under the physical measure, an arithmetic LS process

$$
d x_{t}=\mu d t+\sigma d L_{t}^{L S}
$$

where $d L_{t}^{L S} \sim S_{\alpha}\left(d t^{1 / \alpha}, \beta, 0\right)$ is the increment of an $L S$ process with $0<\alpha \leq 2,-1 \leq \beta \leq 1$, $\sigma>0$ and $\mu$ is a constant. Then the value of a European-style option with final payoff $\Pi\left(x_{T}, T\right)$ satisfies the following fractional differential equation

$$
\begin{aligned}
r V(x, t)= & \frac{\partial V(x, t)}{\partial t}+\left(r-\beta \sigma^{\alpha} \sec (\alpha \pi / 2)\right) \frac{\partial V(x, t)}{\partial x}-\kappa_{2}^{\alpha} \sec (\alpha \pi / 2){ }_{-\infty} D_{x}^{\alpha} V(x, t) \\
& +\kappa_{1}^{\alpha} \sec (\alpha \pi / 2)\left(V(x, t)-e^{x}{ }_{x} D_{\infty}^{\gamma} e^{-x} V(x, t)\right),
\end{aligned}
$$

where

$$
\kappa_{2}^{\alpha}=\frac{1-\beta}{2} \sigma^{\alpha} \quad \text { and } \quad \kappa_{1}^{\alpha}=\frac{1+\beta}{2} \sigma^{\alpha}
$$

For a proof see Appendix D.

Note that the case when $\alpha=2$ and $\beta=0$ yields

$$
\begin{aligned}
r V(x, t) & =\frac{\partial V(x, t)}{\partial t}+\left(r-\sigma^{2}\right) \frac{\partial V(x, t)}{\partial x}+\frac{1}{2} \sigma^{2}{ }_{-\infty} D_{x}^{2} V(x, t)+\frac{1}{2} \sigma^{2}{ }_{x} D_{\infty}^{2} V(x, t) \\
& =\frac{\partial V(x, t)}{\partial t}+\left(r-\sigma^{2}\right) \frac{\partial V(x, t)}{\partial x}+\sigma^{2} \frac{\partial^{2} V(x, t)}{\partial x^{2}}
\end{aligned}
$$

which is the classical Black-Scholes partial differential equation in $x_{t}=\ln S_{t}$. Note also that given the parametrisation of the LS distribution we use here, when $X \sim S_{2}(\sigma, 0,0)$, the expected value $\mathbb{E}\left[X^{2}\right]=2 \sigma^{2}$. This is the reason why the constant coefficients show $\sigma^{2}$ instead of the usual $\sigma^{2} / 2$ in the classical Black-Scholes operator shown above. 
Another very important case is when $\alpha>1$ and $\beta=-1$; this is known as the FMLS process of Carr and Wu (2003a).

European-style options written on an underlying that follows the risk-neutral process (D9) with $\beta=-1$

$$
d\left(\ln S_{t}\right)=\left(r+\sigma^{\alpha} \sec (\alpha \pi / 2)\right) d t+\sigma d \tilde{L}_{t}^{L S}
$$

satisfy the FMLS FBS equation

$$
r V(x, t)=\frac{\partial V(x, t)}{\partial t}+\left(r+\sigma^{\alpha} \sec (\alpha \pi / 2)\right) \frac{\partial V(x, t)}{\partial x}-\sigma^{\alpha} \sec (\alpha \pi / 2){ }_{-\infty} D_{x}^{\alpha} V(x, t) .
$$

Moreover, to derive the corresponding FBS equation, when the risk-neutral dynamics of the stock price are driven by a CGMY process, we proceed as above. The stock dynamics are given by

$$
d x_{t}=\left(r-w_{c g m y}\right) d t+d \tilde{L}_{t}^{C G M Y}
$$

where $x_{t}=\ln S_{t}$ and

$$
w_{\text {cgmy }}=C \Gamma(Y)\left\{(M-1)^{Y}-M^{Y}+(G+1)^{Y}-G^{Y}\right\} .
$$

Hence the CGMY FBS is given by

$$
\begin{aligned}
\frac{\partial V(x, t)}{\partial t}= & \left(r+\sigma\left(M^{Y}+G^{Y}\right)\right) V(x, t)-\left(r-w_{c g m y}\right) \frac{\partial V(x, t)}{\partial x} \\
& -\sigma\left[e^{M x}{ }_{-\infty} D_{x}^{Y}\left(e^{-M x} V(x, t)\right)+e^{-G x}{ }_{x} D_{\infty}^{Y}\left(e^{G x} V(x, t)\right)\right],
\end{aligned}
$$

where $\sigma=C \Gamma(-Y)$. 
Note that $Y<2$, so that in the case where $Y<0$ the fractional operators shown in (27) are in fact fractional integrals as shown in Definition 1. Moreover, we also need the condition $M \geq 1$ to be satisfied so that $\mathbb{E}_{t}\left[S_{T}\right]<\infty$.

Finally, for the DL or KoBoL process we proceed as above to obtain the corresponding FBS equation; see (D13) in the appendix for details.

\section{Conclusions}

This paper shows that the calculus of fractional operators is related to some of the most important jump processes used in the financial literature, for example the FMLS, KoBoL, Koponen's DL and CGMY. More importantly, we have devised a dynamic hedging strategy based on fractional operators and tested it for different models. We have compared our proposed Delta-Fractional-Gamma hedging strategy with the well-known approach of Delta-Gammaneutrality and looked in detail at simulations under the FMLS, LS and MJD models. We have seen that due to the large movements or jumps in the underlying stock price, fractional operators provide a much better hedge than the traditional Delta- and Gamma-neutral approach. It was argued that since fractional operators take into account information about the value and curvature of the portfolio for a range of the stock price between zero and the current stock price, ie $\left[0, S_{t}\right]$, hedging strategies will perform better according to metrics such as the range in which the P\&L lies, or when applicable, the volatility of the P\&L. We showed that in cases such as the MJD and the FMLS the volatility of the P\&L is within the range of $25 \%$ to $30 \%$ of that resulting from employing Delta- and Gamma-neutrality. Moreover, in very extreme cases such as the LS model, where under the physical measure the log-stock price (due to the heavy tails of the distribution of the underlying uncertainty) exhibits infinite variance, we showed that the fractional strategies considerably reduce the exposure of the P\&L to large shocks. For example, the results from Delta- and Gamma-hedging resulted in P\&L values ranging between 
$[-101,630]$ whereas the results in the same simulation when fractional hedging strategies are applied ranged between $[-5,7]$. 


\section{References}

Black, Fisher, and Myron Scholes, 1973, The pricing of options and corporate liabilities, Journal of Political Economy 81, 637-654.

Boyarchenko, Svetlana, and Sergei Levendorskii, 2000, Option pricing for Truncated Lévy processes, International Journal of Theoretic and Applied Finance 3, 549-552.

Carr, Peter, Helyette Geman, Dilip Madan, and Marc Yor, 2002, The fine Structure of asset returns: an empirical investigation, Journal of Business 75, 305-332.

Carr, Peter, and Dilip Madan, 1999, Option Valuation Using the Fast Fourier Transform, Journal of Computational Finance 2, 61-73.

Carr, Peter, and Liuren Wu, 2003a, The Finite Moment Logstable Process and Option Pricing, The Journal of Finance LVIII, 753-777.

Carr, Peter, and Liuren Wu, 2003b, What Type of Process Underlies Options? A Simple Robust Test, The Journal of Finance VIII, 2581-2410.

Carr, Peter, and Liuren Wu, 2004, Time-Changed Lévy Processes and Option Pricing, Journal of Financial Economics 71, 113-141.

Cont, Rama, and Peter Tankov, 2004, Financial Modelling With Jump Processes. (Chapman and Hall London) 1 st edn.

Feller, William, 1966, An Introduction to Probability Theory and its Applications vol. II. (Wiley).

Heston, Steven L., 1993, A Closed-Form Solution for Options with Stochastic Volatility with Applications to Bond and Currency Options, The Review of Financial Studies 6, 327-343.

Koponen, Ismo, 1995, Analytic Approach to the Problem of Convergence of Truncated Levy Flights towards the Gaussian Stochastic Process, Physical Review E 52, 1197-1199.

Kou, Steve G., 2002, A Jump-Diffusion Model for Option Pricing, Management Science 48, 10861101. 
Lewis, Alan, 2001, A Simple Option Formula for General Jump-Diffusion and other Exponential Lévy Processes, Working Paper.

Madan, Dilip, and Eugene Seneta, 1990, The Variance Gamma (VG) model for share market returns, Journal of Business 63, 511-524.

Mandelbrot, Benoit, 1997, Fractals and Scaling in Finance. (Springer) 1st edn.

Mantegna, Rosario N., and H. Eugene Stanley, 2000, An Introduction to Econophysics, correlations and complexity in finance. (Cambridge) 1 st edn.

Matacz, Andrew, 2000, Financial Modeling and Option Theory with the Truncated Levy Process, International Journal of Theoretical and Applied Finance 3, 143-160.

McCulloch, J. Huston, 2003, The Risk-Neutral Measure under Log-Stable Uncertainty, Working Paper.

Merton, Robert, 1990, Continuous-Time Finance. (Blackwell) 1st edn.

Miller, Kenneth S., and Bertram Ross, 1993, An Introduction to the Fractional Calculus and Fractional Differential Equations. (John Wiley and Sons New York) 1st edn.

Podlubny, Igor, 1999, Fractional Differential Equations vol. 198 of Mathematics in Science and Engineering. (Academic Press San Diego, California) 1st edn.

Samko, Stefan G., Anatoly A. Kilbas, and Oleg I. Marichev, 1993, Fractional Integrals and Derivatives, theory and applications. (Gordon and Breach Science Publishers).

Samorodnitsky, Gennady, and Murad Taqqu, 1994, Stable Non-Gaussian Random Processes, Stochastic Models with Infinite Variance. (Chapman Hall) 1st edn.

Schoutens, Wim, 2003, Lévy Processes in Finance. (Wiley England) 1st edn.

Stein, Elias M., and Jeremy C. Stein, 1991, Stock Price Distributions with Stochastic Volatility: An Analytic Approach, The Review of Financial Studies 4, 727-752. 


\section{Appendix A. Lévy process}

Proposition 4 Lévy-Khintchine representation. Let $X_{t}$ be a Lévy process. Then the natural logarithm of the characteristic function can be written as

$$
\ln \mathbb{E}\left[e^{i \xi X_{t}}\right]=c i t \xi-\frac{1}{2} d^{2} t \xi^{2}+t \int_{\mathbb{R} \backslash\{0\}}\left(e^{i \xi x}-1-i \xi x \mathbf{I}_{|x|<1}\right) W(d x),
$$

where $c \in \mathbb{R}, d \geq 0, \xi \in \mathbb{C}, \mathbf{I}$ is the indicator function and the Lévy measure $W$ must satisfy

$$
\int_{\mathbb{R}} \min \left\{1, x^{2}\right\} W(d x)<\infty
$$

A Lévy process can be seen as a combination of a drift component, a Brownian motion (Gaussian) component and a jump component. These three components are determined by the Lévy-Khintchine triplet $\left(c, d^{2}, W\right)$. The parameter $c$ parametrises the 'trend' component which is responsible for the development of the process $X_{t}$ on the average. The parameter $d^{2}$ defines the variance of the continuous Gaussian component of $X_{t}$. The Lévy measure $W$ is responsible for the behaviour of the jump component of $X_{t}$ and determines the frequency and magnitude of jumps. Finally, if the Lévy measure is of the form $W(d x)=w(x) d x$, we call $w(x)$ the Lévy density, which measures the arrival rate of the jumps of the underlying process $x$. We note that in (A1) above we can have different centering functions; that is, instead of having the term $i \xi x \mathbf{I}_{|x|<1}$, we may have other functional forms that guarantee integrability around zero. For example we could simply choose to have $i \xi x$ (ie without the indicator function) and the difference in the Lévy-Khintchine representation will be in the drift component. Moreover, for

some types of processes, like the CGMY, it is sufficient to have, $\int_{-\infty}^{\infty}\left(e^{i \xi x}-1\right) W(d x)$ for the jump part of the process, see Carr, Geman, Madan, and Yor (2002).

\section{Appendix B. Fractional Derivatives}

In this section we depict fractional derivatives for European call options using different assumptions for the stochastic process followed by the underlying security $S_{t}$. 


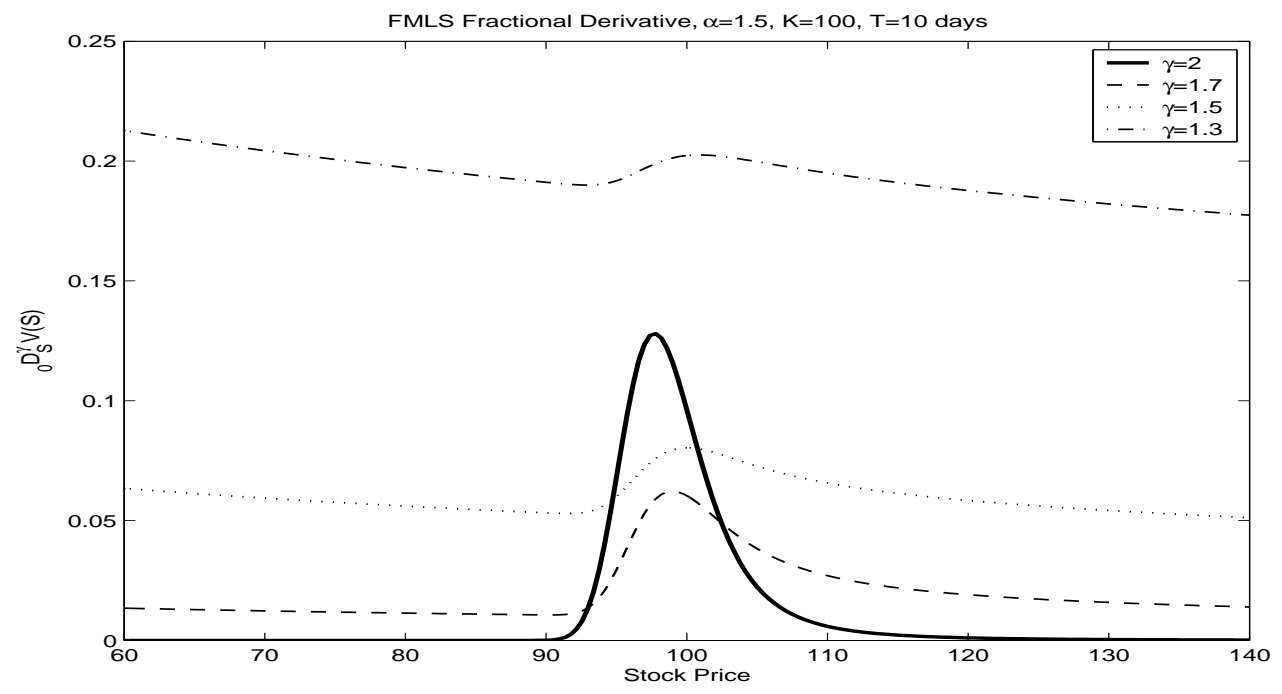

Figure 10. Fractional Derivative for FMLS. The figure shows fractional derivatives ${ }_{0} D_{S}^{\gamma} V(S, t ; K, T)$ with $\gamma=2, \gamma=1.7, \gamma=1.5$ and $\gamma=1.3$ for a European call with $S_{0}=100$, $K=100$ and $T=10$ days to expiry when the underlying follows, under the risk-neutral measure, an FMLS process with $\sigma=0.2, \alpha=1.5$ and drift $\mu=0.05$.

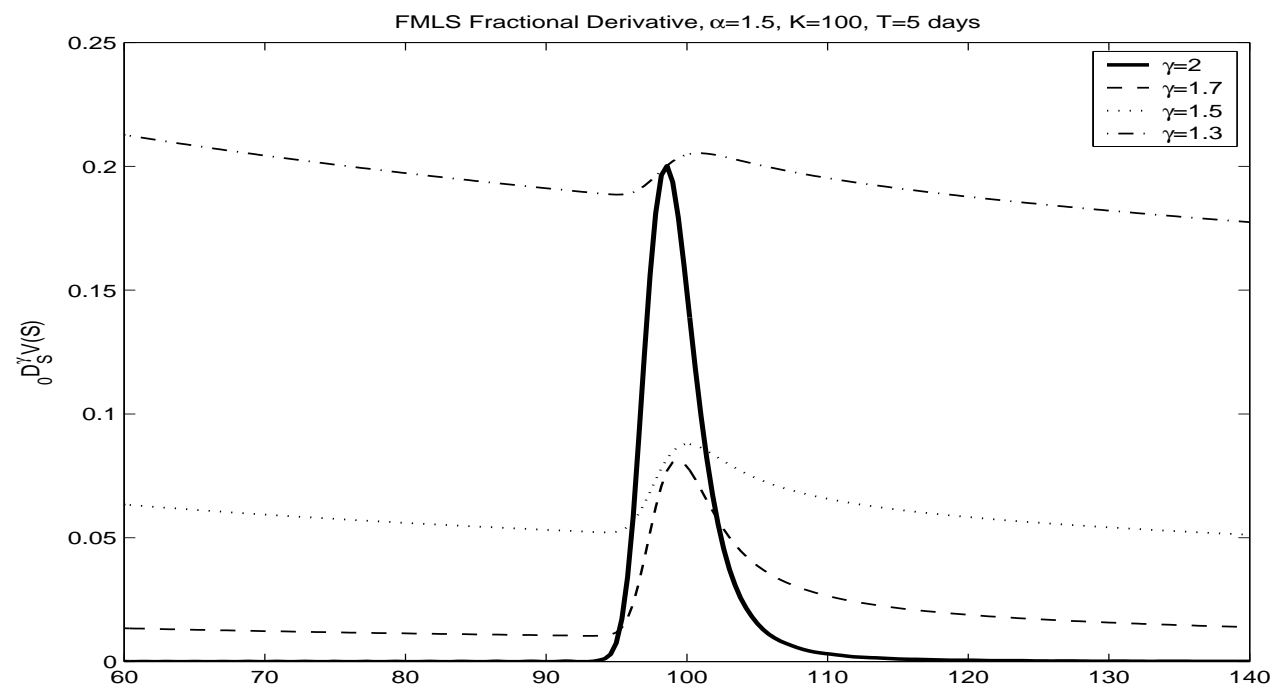

Figure 11. Fractional Derivative for FMLS. The figure shows fractional derivatives ${ }_{0} D_{S}^{\gamma} V(S, t ; K, T)$ with $\gamma=2, \gamma=1.7, \gamma=1.5$ and $\gamma=1.3$ for a European call with $S_{0}=100$, $K=100$ and $T=5$ days to expiry when the underlying follows, under the risk-neutral measure, an FMLS process with $\sigma=0.2, \alpha=1.5$ and drift $\mu=0.05$ 


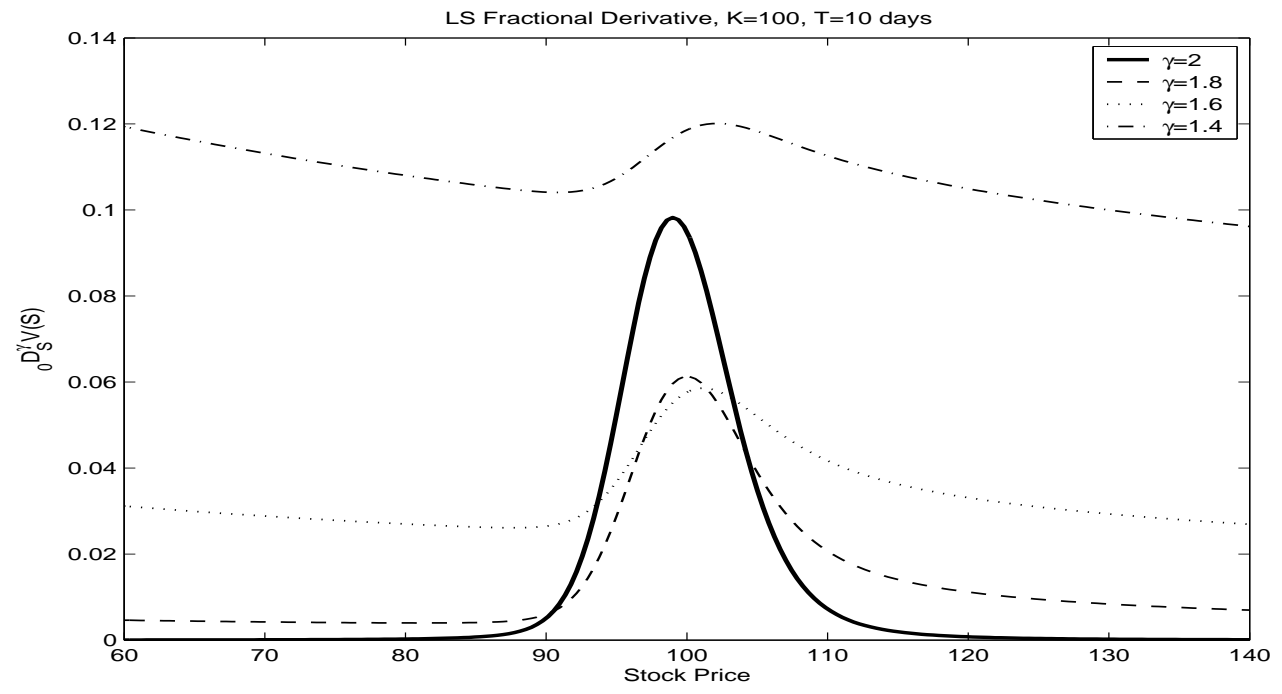

Figure 12. Fractional Derivative for LS. The figure shows fractional derivatives ${ }_{0} D_{S}^{\gamma} V(S, t ; K, T)$ with $\gamma=2, \gamma=1.8, \gamma=1.6$ and $\gamma=1.4$ for a European call with $S_{0}=100$, $K=100$ and $T=10$ days to expiry when the underlying follows, under the risk-neutral measure, an LS process with $\sigma=0.2, \alpha=1.7, \beta=-0.5$ and drift $\mu=0.05$.

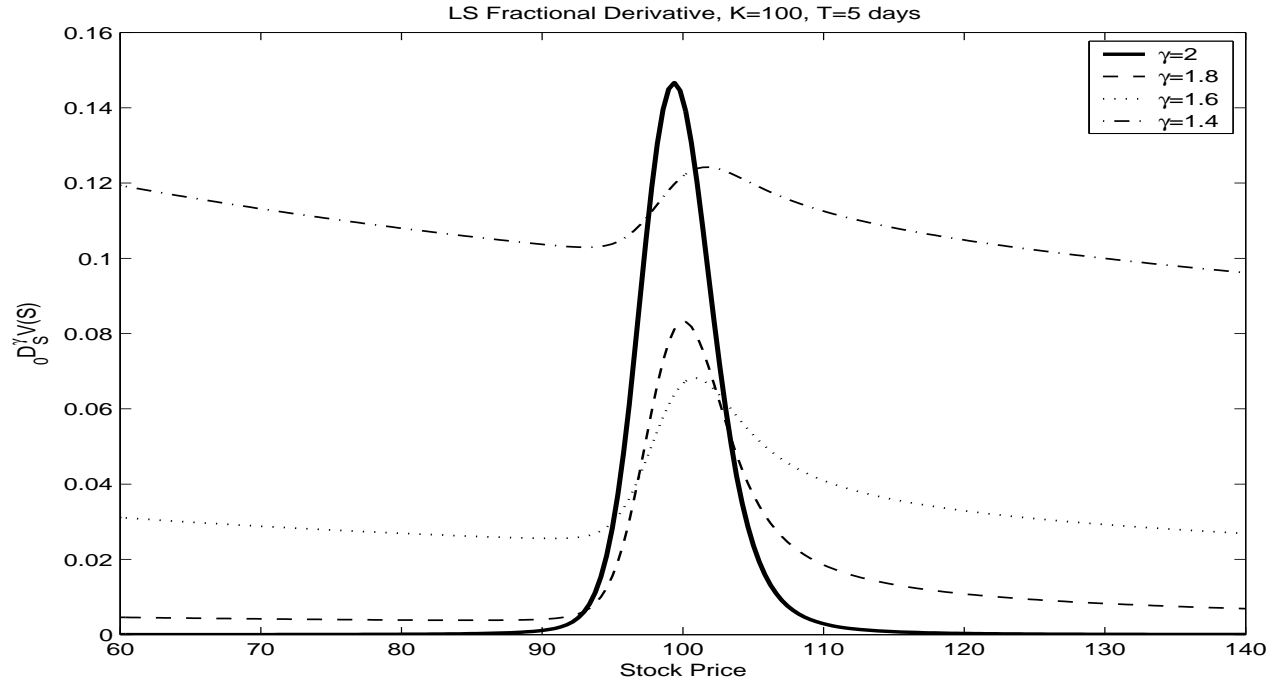

Figure 13. Fractional Derivative for LS. The figure shows fractional derivatives ${ }_{0} D_{S}^{\gamma} V(S, t ; K, T)$ with $\gamma=2, \gamma=1.8, \gamma=1.6$ and $\gamma=1.4$ for a European call with $S_{0}=100$, $K=100$ and $T=5$ days to expiry when the underlying follows, under the risk-neutral measure, an LS process with $\sigma=0.2, \alpha=1.7, \beta=-0.5$ and drift $\mu=0.05$ 


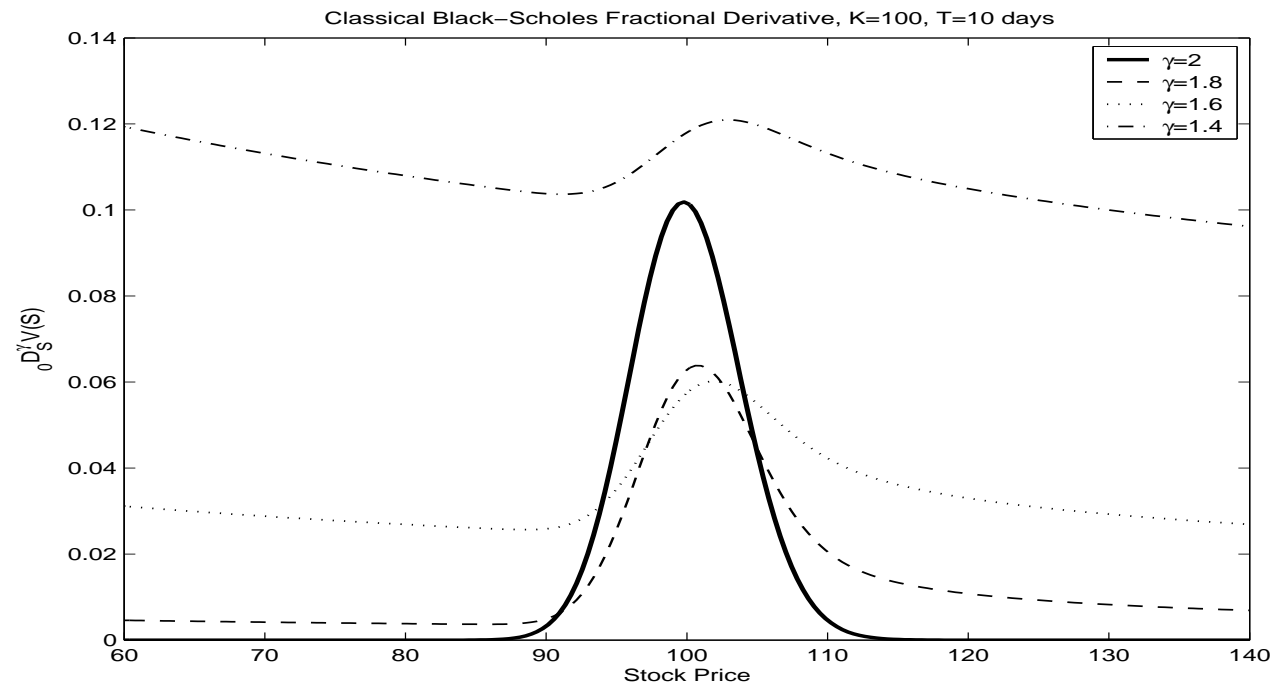

Figure 14. Fractional Derivative for Classical Black-Scholes. The figure shows fractional derivatives ${ }_{0} D_{S}^{\gamma} V(S, t ; K, T)$ with $\gamma=2, \gamma=1.8, \gamma=1.6$ and $\gamma=1.4$ for a European call with $S_{0}=100, K=100$ and $T=10$ days to expiry when the underlying follows, under the riskneutral measure, a geometric Brownian motion with volatility $\sigma=0.2$, and drift $\mu=0.05$.

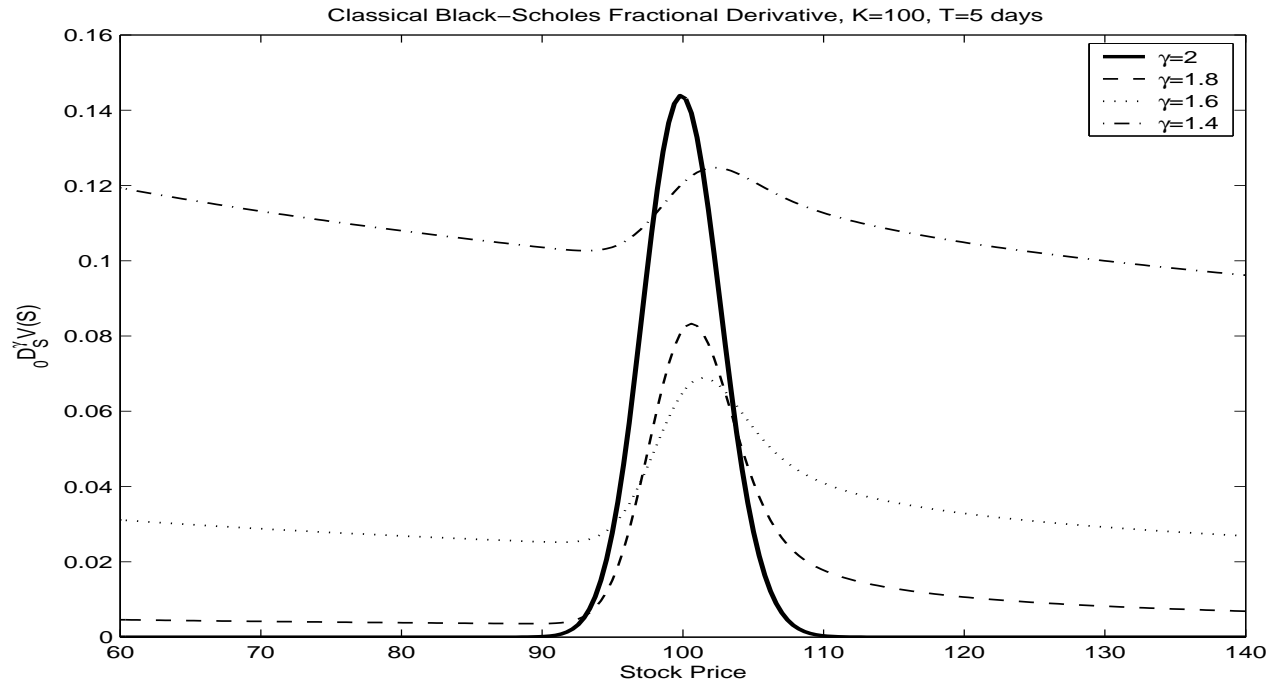

Figure 15. Fractional Derivative for Classical Black-Scholes. The figure shows fractional derivatives ${ }_{0} D_{S}^{\gamma} V(S, t ; K, T)$ with $\gamma=2, \gamma=1.8, \gamma=1.6$ and $\gamma=1.4$ for a European call with $S_{0}=100, K=100$ and $T=5$ days to expiry when the underlying follows, under the riskneutral measure, a geometric Brownian motion with volatility $\sigma=0.2$, and drift $\mu=0.05$. 


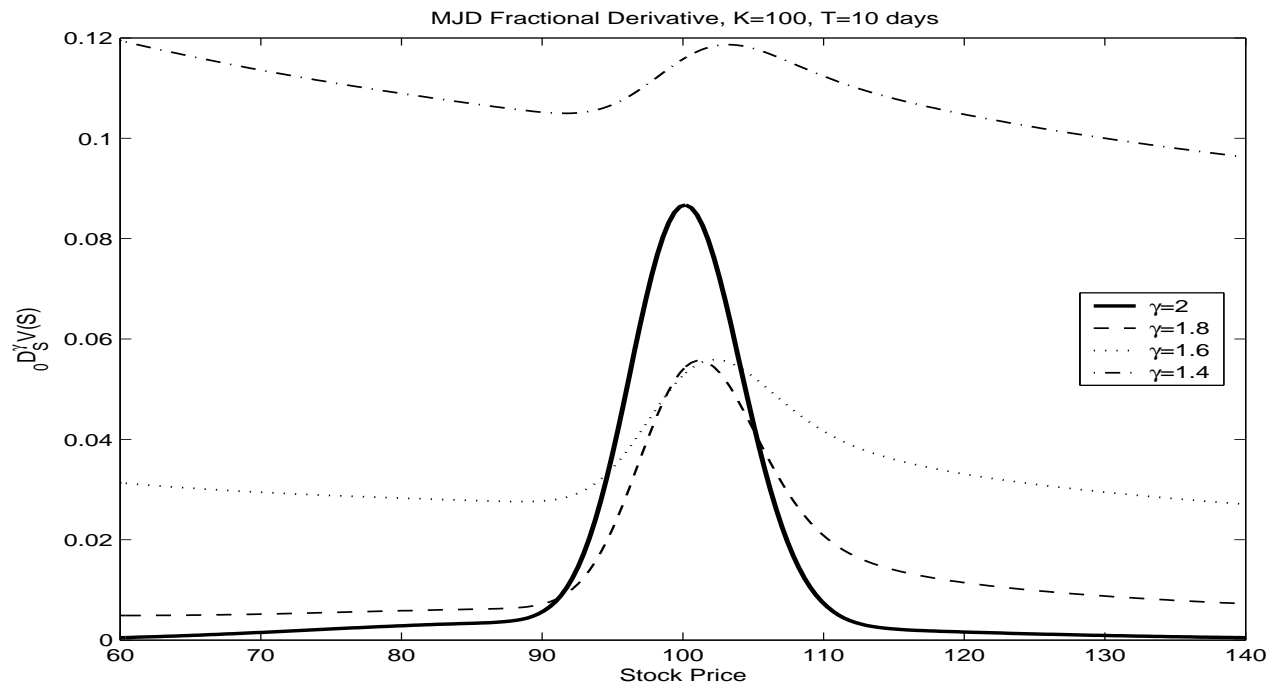

Figure 16. Fractional Derivative for MJD. The figure shows fractional derivatives ${ }_{0} D_{S}^{\gamma} V(S, t ; K, T)$ with $\gamma=2, \gamma=1.8, \gamma=1.6$ and $\gamma=1.4$ for a European call with $S_{0}=100$, $K=100$ and $T=10$ days to expiry when the underlying follows, under the risk-neutral measure, a MJD process with $\sigma=0.2, \lambda=5, \sigma_{J}=0.2$ and $\mu_{J}=0$.

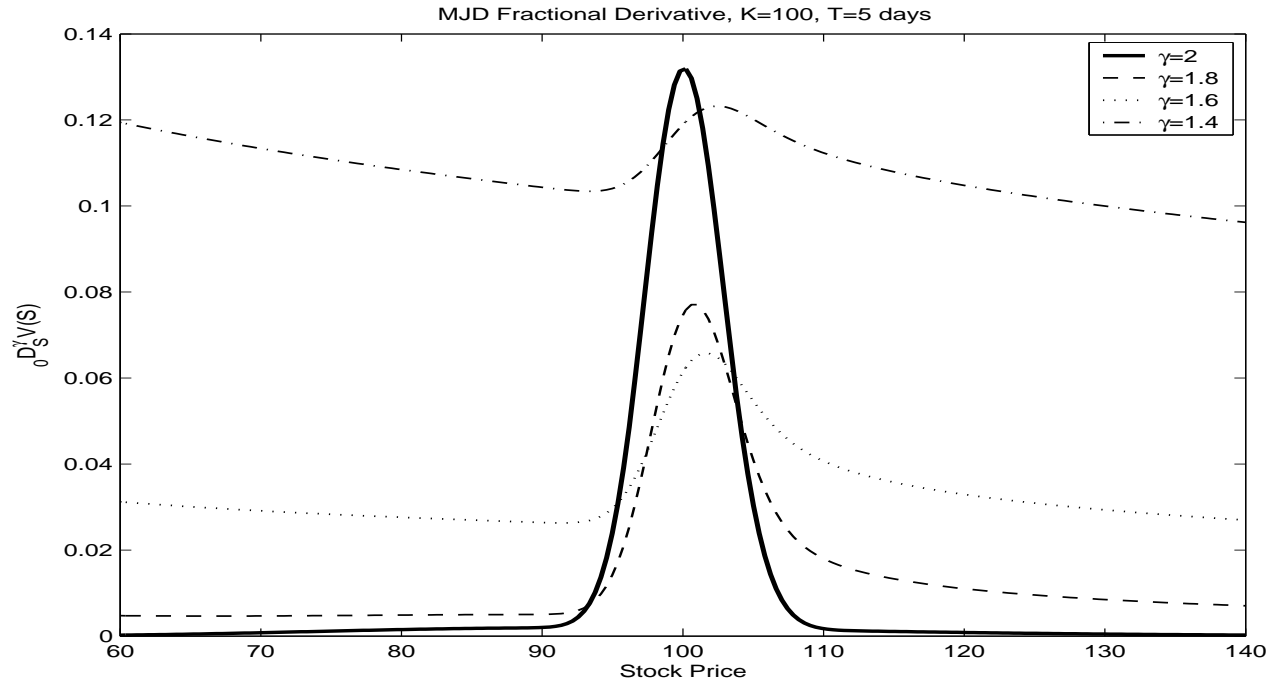

Figure 17. Fractional Derivative for MJD. The figure shows fractional derivatives ${ }_{0} D_{S}^{\gamma} V(S, t ; K, T)$ with $\gamma=2, \gamma=1.8, \gamma=1.6$ and $\gamma=1.4$ for a European call with $S_{0}=100$, $K=100$ and $T=10$ days to expiry when the underlying follows, under the risk-neutral measure, a MJD process with $\sigma=0.2, \lambda=5, \sigma_{J}=0.2$ and $\mu_{J}=0$. 


\section{Appendix C. Calculation of fractional derivatives in the com- plex plane}

In the fractional Taylor's expansion we use derivatives of the form

$$
{ }_{0} D_{S}^{\gamma} V(S, t)=\frac{1}{\Gamma(n-\gamma)} \frac{d^{n}}{d S^{n}} \int_{0}^{S}(S-y)^{n-1-\gamma} V(y, t) d y
$$

We calculate these derivatives by numerically inverting their Fourier transforms; these are given in the following proposition.

Proposition 5 Let $1<\gamma<2$. Then

$$
\mathcal{F}\left\{{ }_{0} D_{S}^{\gamma} V(S, t)\right\}=\mathcal{F}\left\{\left.\frac{S^{1-\gamma}}{\Gamma(2-\gamma)} \frac{d V(S, t)}{d S}\right|_{S=0}\right\}+\frac{\Gamma(-i \xi-1+\gamma)}{\Gamma(-i \xi-1)} \hat{V}(\xi-i(2-\gamma), t)
$$

Proof: First we use integration by parts to write

$$
{ }_{0} D_{S}^{\gamma} V(S, t)=\left.\frac{1}{\Gamma(2-\gamma)} \frac{d V(S, t)}{d S}\right|_{S=0}+\frac{1}{\Gamma(2-\gamma)} \int_{0}^{S}(S-y)^{1-\gamma} \frac{d^{2} V(y, t)}{d y^{2}} d y
$$

Now let us focus on the second term on the right-hand side of the equation above substitute $y=u S$ to obtain

$$
\frac{1}{\Gamma(2-\gamma)} \int_{0}^{S}(S-y)^{1-\gamma} \frac{d^{2} V(y, t)}{d y^{2}} d y=\frac{S^{2-\gamma}}{\Gamma(2-\gamma)} \int_{0}^{1}(1-u)^{1-\gamma} \frac{d^{2} V(u S, t)}{d u^{2}} d u
$$

Now let's take the Fourier transform with respect to the stock price $S=e^{x}$.

$$
\mathcal{F}\left\{\frac{S^{2-\gamma}}{\Gamma(2-\gamma)} \int_{0}^{1}(1-u)^{1-\gamma} \frac{d^{2}}{d u^{2}} V(u S, t) d u\right\}=\frac{1}{\Gamma(2-\gamma)} \int_{0}^{1}(1-u)^{1-\gamma} \frac{d^{2}}{d u^{2}} \mathcal{F}\left\{e^{(2-\gamma) x} V\left(u e^{x}, t\right)\right\} d u
$$




$$
\begin{aligned}
\mathcal{F}\left\{e^{(2-\gamma) x} V\left(u e^{x}, t\right)\right\} & =\int_{-\infty}^{\infty} e^{i \xi x} e^{(2-\gamma) x} V\left(e^{x+\ln u}, t\right) d x, \quad \text { now let } m=x+\ln u \\
& =\int_{-\infty}^{\infty} e^{(i \xi+2-\gamma)(m-\ln u)} V\left(e^{m}, t\right) d m \\
& =e^{-(i \xi+2-\gamma) \ln u} \int_{-\infty}^{\infty} e^{i(\xi-i(2-\gamma)) m} V\left(e^{m}, t\right) d m \\
& =e^{-(i \xi+2-\gamma) \ln u} \hat{V}(\xi-i(2-\gamma), t) .
\end{aligned}
$$

Combining the results together we obtain

$$
\begin{aligned}
\mathcal{F}\left\{\frac{S^{2-\gamma}}{\Gamma(2-\gamma)} \int_{0}^{1}(1-u)^{1-\gamma} \frac{d^{2} V(u S, t)}{d u^{2}} d u\right\} & =\frac{1}{\Gamma(2-\gamma)} \int_{0}^{1}(1-u)^{1-\gamma} \frac{d^{2}}{d u^{2}} e^{-(i \xi+2-\gamma) \ln u \hat{V}(\xi-i(2-\gamma), t) d u} \\
& =\frac{1}{\Gamma(2-\gamma)} \int_{0}^{1}(1-u)^{1-\gamma} \frac{d^{2}}{d u^{2}} u^{-(i \xi+2-\gamma)} \hat{V}(\xi-i(2-\gamma), t) d u \\
& =\frac{(i \xi+2-\gamma)(i \xi+3-\gamma)}{\Gamma(2-\gamma)} \int_{0}^{1}(1-u)^{1-\gamma} u^{-(i \xi+4-\gamma)} \hat{V}(\xi-i(2-\gamma), t) d u \\
& =\frac{(i \xi+2-\gamma)(i \xi+3-\gamma)}{\Gamma(2-\gamma)} \hat{V}(\xi-i(2-\gamma), t) \int_{0}^{1}(1-u)^{1-\gamma} u^{-(i \xi+4-\gamma)} d u \\
& =\frac{(i \xi+2-\gamma)(i \xi+3-\gamma)}{\Gamma(2-\gamma)} \hat{V}(\xi-i(2-\gamma), t) \mathrm{B}(-i \xi-3+\gamma, 2-\gamma) \\
& =\frac{(i \xi+2-\gamma)(i \xi+3-\gamma)}{\Gamma(2-\gamma)} \frac{\Gamma(-i \xi-3+\gamma) \Gamma(2-\gamma)}{\Gamma(-i \xi-1)} \hat{V}(\xi-i(2-\gamma), t) \\
& =(i \xi+2-\gamma)(i \xi+3-\gamma) \frac{\Gamma(-i \xi-3+\gamma)}{\Gamma(-i \xi-1)} \hat{V}(\xi-i(2-\gamma), t) \\
& =-(i \xi+2-\gamma) \frac{\Gamma(-i \xi-2+\gamma)}{\Gamma(-i \xi-1)} \hat{V}(\xi-i(2-\gamma), t) \\
& =\frac{\Gamma(-i \xi-1+\gamma)}{\Gamma(-i \xi-1)} \hat{V}(\xi-i(2-\gamma), t),
\end{aligned}
$$

where $\mathrm{B}(w, z)=\Gamma(w) \Gamma(z) / \Gamma(z+w)$ is the Beta function and we have used $\Gamma(z+1)=z \Gamma(z)$.

Note that for $\gamma=2$ we obtain $\left((i \xi)^{2}+i \xi\right) \hat{V}(\xi, t)$, which is the Fourier transform of $S^{2} V_{S S}(S, t)$. 


\section{Appendix D. Other propositions and proofs}

Proof of Proposition 2

The value of the option satisfies

$$
V(x, t)=e^{-r(T-t)} \mathbb{E}_{t}\left[\Pi\left(x_{T}, T\right)\right] .
$$

Now assume that the payoff $\Pi\left(x_{T}, T\right)$ has a complex Fourier transform (CFT), denoted by a circumflex,

$$
\mathcal{F}\left\{\Pi\left(x_{T}, T\right)\right\} \equiv \hat{\Pi}(\xi, T)=\int_{-\infty+i \xi_{i}}^{\infty+i \xi_{i}} e^{i \xi x_{T}} \Pi\left(x_{T}, T\right) d x_{T}
$$

in the strip $a<\xi_{i}<b$, where we denote $\xi_{i}=\operatorname{Im} \xi$. Then we can write

$$
V(x, t)=\frac{e^{-r(T-t)}}{2 \pi} \mathbb{E}_{t}\left[\int_{-\infty+i \xi_{i}}^{\infty+i \xi_{i}} e^{-i x_{T} \xi} \hat{\Pi}(\xi, T) d \xi\right]
$$

Now taking the expectation operator inside the integral, see Lewis (2001), we obtain

$$
\begin{aligned}
V(x, t) & =\frac{e^{-r(T-t)}}{2 \pi} \int_{-\infty+i \xi_{i}}^{\infty+i \xi_{i}} \mathbb{E}_{t}\left[e^{-i x_{T} \xi}\right] \hat{\Pi}(\xi, T) d \xi \\
& =\frac{e^{-r(T-t)}}{2 \pi} \int_{-\infty+i \xi_{i}}^{\infty+i \xi_{i}} e^{-i \xi x_{t}-i \xi \mu(T-t)} e^{(T-t) \Psi(-\xi)} \hat{\Pi}(\xi, T) d \xi
\end{aligned}
$$

where $e^{\Psi(\xi)}$ is the characteristic function of $\sigma \int_{0}^{1} d L_{s}$. Note that we require $e^{\Psi(-\xi)}$ to be analytic in a strip that intersects the strip where the CFT of the payoff exists.

It is straightforward to see that (D5) can be written as

$$
\frac{1}{2 \pi} \int_{-\infty+i \xi_{i}}^{\infty+i \xi_{i}} e^{-i \xi x_{t}} \hat{V}(\xi, t) d \xi=\frac{e^{-r(T-t)}}{2 \pi} \int_{-\infty+i \xi_{i}}^{\infty+i \xi_{i}} e^{-i \xi x_{t}-i \xi \mu(T-t)} e^{(T-t) \Psi(-\xi)} \hat{\Pi}(\xi, T) d \xi
$$

and, by applying the Fourier transform $\mathcal{F}$ to both sides of equation (D6), we obtain

$$
\hat{V}(\xi, t)=e^{-r(T-t)} e^{-i \xi \mu(T-t)} e^{(T-t) \Psi(-\xi)} \hat{\Pi}(\xi, T) .
$$


Reordering terms, and taking the principal value of the logarithm function, yields

$$
\ln (\hat{V}(\xi, t) / \hat{\Pi}(\xi, T))=-r(T-t)-i \xi \mu(T-t)+(T-t) \Psi(-\xi)
$$

Now, differentiating with respect to time $(\partial / \partial t)$, we note that (D7) is the solution of the ordinary differential equation (ODE)

$$
\frac{\partial \hat{V}(\xi, t)}{\partial t}=[r+i \xi \mu-\Psi(-\xi)] \hat{V}(\xi, t)
$$

with boundary condition $\hat{V}(\xi, T)=\hat{\Pi}(\xi, T)$.

Moreover, note that we can use this ODE to find the integro-differential equation satisfied by the value of options written on a stock that follows a geometric Lévy process. For simplicity let us assume that the Lévy triplet is $(0,0, W)$, ie that it has no location and no Gaussian component. Then the next step is to apply the inverse Fourier transform $\mathcal{F}^{-1}$ to the ODE above. Thus

$$
\begin{aligned}
\frac{\partial V(x, t)}{\partial t}= & \frac{1}{2 \pi} \int_{-\infty+i \xi_{i}}^{\infty+i \xi_{i}} e^{-i \xi x_{t}}[r+i \xi \mu-\Psi(-\xi)] \hat{V}(\xi, t) d \xi \\
= & \frac{1}{2 \pi} \int_{-\infty+i \xi_{i}}^{\infty+i \xi_{i}} e^{-i \xi x_{t}}(r+i \xi \mu) \hat{V}(\xi, t) d \xi-\frac{1}{2 \pi} \int_{-\infty+i \xi_{i}}^{\infty+i \xi_{i}} e^{-i \xi x_{t}} \Psi(-\xi) \hat{V}(\xi, t) d \xi \\
= & r V(x, t)-\mu \frac{\partial V(x, t)}{\partial x} \\
& -\frac{1}{2 \pi} \int_{-\infty+i \xi}^{\infty+i \xi_{i}} e^{-i \xi x_{t}} \int_{-\infty}^{\infty}\left(e^{-i \xi y}-1+i \xi y \mathbf{I}_{|y|<1}\right) W(d y) \hat{V}(\xi, t) d \xi \\
= & r V(x, t)-\mu \frac{\partial V(x, t)}{\partial x} \\
& -\frac{1}{2 \pi} \int_{-\infty}^{\infty} \int_{-\infty+i \xi_{i}}^{\infty+i \xi_{i}} e^{-i \xi x_{t}}\left(e^{-i \xi y}-1+i \xi y \mathbf{I}_{|y|<1}\right) \hat{V}(\xi, t) d \xi W(d y) \\
= & r V(x, t)-\mu \frac{\partial V(x, t)}{\partial x} \\
& -\int_{-\infty}^{\infty}\left(V(x+y, t)-V(x, t)-y \frac{\partial V(x, t)}{\partial x} \mathbf{I}_{|y|<1}\right) W(d y) .
\end{aligned}
$$

Note that by applying Fubini's theorem we can interchange the order of integration to obtain (D8). 


\section{Proof of Proposition 3}

McCulloch (2003) showed that the corresponding risk-neutral process is given by

$$
d\left(\ln S_{t}\right)=\left(r-\beta \sigma^{\alpha} \sec (\alpha \pi / 2)\right) d t+\sigma d \tilde{L}_{t}^{L S}+\sigma d \tilde{L}_{t}^{D L}
$$

where $d \tilde{L}_{t}^{L S}$ and $d \tilde{L}_{t}^{D L}$ are independent and are the increments of a maximally negatively skewed LS process and the increment of a maximally positively skewed DL process, ie.

$$
\sigma \tilde{L}_{1}^{L S} \sim S_{\alpha}\left(\kappa_{2},-1,0\right) \quad \text { and } \quad \sigma \tilde{L}_{1}^{D L} \sim D L_{\alpha}\left(\kappa_{1}, 1,0,0,1\right)
$$

with $\kappa_{1}$ and $\kappa_{2}$ as in (23).

In this case the ODE (21) becomes

$$
\frac{\partial \hat{V}(\xi, t)}{\partial t}=\left[r+i \xi\left(r-\beta \sigma^{\alpha} \sec (\alpha \pi / 2)\right)-\Psi_{L S}(-\xi)-\Psi_{D L}(-\xi)\right] \hat{V}(\xi, t)
$$

where

$$
\Psi_{L S}(-\xi)=\kappa_{2}^{\alpha} \sec (\alpha \pi / 2)(-i \xi)^{\alpha}
$$

and

$$
\Psi_{D L}(-\xi)=\kappa_{1}^{\alpha} \sec (\alpha \pi / 2)\left(1-(1+i \xi)^{\alpha}\right)
$$

Taking the inverse Fourier transform of (D11) delivers the result.

\section{The KoBoL or DL FBS equation}

To obtain the DL or KoBoL FBS equation we assume that the risk-neutral log-stock price dynamics follow a DL process

$$
d x_{t}=\left(r-w_{d l}\right) d t+d L_{t}^{D L}
$$


where

$$
w_{d l}=\kappa^{\alpha}\left\{p(\lambda-1)^{\alpha}+q(\lambda+1)^{\alpha}-\lambda^{\alpha}-\alpha \lambda^{\alpha-1}(q-p)\right\},
$$

$x_{t}=\ln S_{t}$ and $r$ is the risk-free rate. Then, proceeding as above, the value of a European-style option with final payoff $\Pi(x, T)$ satisfies the following FBS equation

$$
\begin{aligned}
\left(r+\kappa^{\alpha} \lambda^{\alpha}\right) V(x, t)= & \frac{\partial V(x, t)}{\partial t}+\left(r-w_{d l}-\lambda^{\alpha-1}(q-p)\right) \frac{\partial V(x, t)}{\partial x} \\
& +\kappa^{\alpha}\left[p e^{\lambda x}{ }_{-\infty} D_{x}^{\alpha} e^{-\lambda x} V(x, t)+q e^{-\lambda x}{ }_{x} D_{\infty}^{\alpha} e^{\lambda x} V(x, t)\right] .
\end{aligned}
$$

Note that if we let $\alpha=2, p=q=1 / 2$ and $\lambda=0$ we obtain the Black-Scholes PDE.

Proposition 6 Let $\vartheta$ be a uniform random variable on $(-\pi / 2, \pi / 2)$ and let $\varepsilon$ be exponential with mean 1. Assume $\vartheta$ and $\varepsilon$ are independent. Then

$$
X=\frac{\sin \alpha \vartheta}{(\cos \vartheta)^{1 / \alpha}}\left(\frac{\cos ((1-\alpha) \vartheta)}{\varepsilon}\right)^{(1-\alpha) / \alpha}
$$

is $S_{\alpha}(1,0,0)$.

For a proof see Samorodnitsky and Taqqu (1994). 


\section{Notes}

${ }^{1}$ Note that a sufficient condition for the integrals (1) and (2) to converge is that

$$
f(x)=O\left(|x|^{-\gamma-\varepsilon}\right) \quad \text { for } \varepsilon>0 \quad \text { as } x \rightarrow \pm \infty .
$$

${ }^{2}$ By large we mean that the movements of the underlying are much larger than those predicted by Gaussian shocks.

${ }^{3}$ Note that the condition on the value of $Y$ is given by (A2).

${ }^{4}$ It is interesting to note that the Lévy density of the CGMY and the DL process is essentially the same as that of the LS process except that the exponential damping factor ensures exponential, instead of polynomial, decay at infinity. We also point out that in the DL case for 'short-time' scales, depending on the magnitude of the damping factor $\lambda$, the distribution of the DL can be seen as a very good approximation to the distribution of the LS, see Matacz (2000).

${ }^{5}$ If we assume that under the physical measure the log-stock price follows a CGMY, DL, KoBoL or FMLS process, then the Esscher transformed process will again be a Lévy process where the corresponding pricing equation can be expressed as a FBS equation.

${ }^{6}$ We note that we do not enquire about the performance of the hedge when $K_{2}<K_{1}$ because the stock price exhibits very large and frequent positive jumps and it is very difficult to implement a Delta- and Gamma-neutral strategy. If simulations were performed, then for a considerable amount of runs, the gamma of $V_{2}\left(S, t ; K_{2}, T_{2}\right)$ would approach zero much faster than that of $V_{1}\left(S, t ; K_{1}, T_{1}\right)$, therefore, in these cases, the hedging strategy would require extremely large amounts, given by

$$
b(S, t)=\frac{\partial^{2} V_{1}(S, t) / \partial S^{2}}{\partial^{2} V_{2}(S, t) / \partial S^{2}},
$$


of $V_{2}\left(S, t ; K_{2}, T_{2}\right)$ to be purchased. 